\title{
Exact equilibrium distributions in statistical quantum field theory with rotation and acceleration: Dirac field
}

\author{
A. Palermo, ${ }^{a, b}$ M. Buzzegoli ${ }^{a, c}$ and F. Becattini ${ }^{a}$ \\ ${ }^{a}$ Dipartimento di Fisica e Astronomia, Università di Firenze and INFN Sezione di Firenze, \\ Via G. Sansone 1, Sesto Fiorentino I-50019, Firenze, Italy \\ ${ }^{b}$ Institut für Theoretische Physik, Johann Wolfgang Goethe-Universität, \\ Max-von-Laue-Str. 1, 60438 Frankfurt am Main, Germany \\ ${ }^{c}$ Department of Physics and Astronomy, Iowa State University, \\ Ames, Iowa 50011, U.S.A. \\ E-mail: andrea.palermo@unifi.it, matteo.buzzegoli@unifi.it, \\ becattini@fi.infn.it
}

ABSTRACT: We derive the general exact forms of the Wigner function, of mean values of conserved currents, of the spin density matrix, of the spin polarization vector and of the distribution function of massless particles for the free Dirac field at global thermodynamic equilibrium with rotation and acceleration, extending our previous results obtained for the scalar field. The solutions are obtained by means of an iterative method and analytic continuation, which lead to formal series in thermal vorticity. In order to obtain finite values, we extend to the fermionic case the method of analytic distillation introduced for bosonic series. The obtained mean values of the stress-energy tensor, vector and axial currents for the massless Dirac field are in agreement with known analytic results in the special cases of pure acceleration and pure rotation. By using this approach, we obtain new expressions of the currents for the more general case of combined rotation and acceleration and, in the pure acceleration case, we demonstrate that they must vanish at the Unruh temperature.

Keywords: Thermal Field Theory, Boundary Quantum Field Theory, Space-Time Symmetries

ArXiv EPrint: 2106.08340 


\section{Contents}

1 Introduction 1

2 Basics of the free Dirac field 3

3 The covariant Wigner function of the free Dirac field $\quad 7$

4 Exact Wigner function at global thermodynamic equilibrium $\quad 8$

4.1 Expectation values $\quad 9$

4.2 The Wigner function 11

$\begin{array}{lll}5 & \text { Currents at global thermodynamic equilibrium } & 13\end{array}$

6 Fermionic analytic distillation $\quad 16$

7 Exact mean values of currents at global thermodynamic equilibrium 20

$\begin{array}{ll}7.1 \text { Acceleration } & 20\end{array}$

$\begin{array}{lll}7.2 & \text { Rotation } & 22\end{array}$

7.3 Thermodynamic equilibrium with rotation and acceleration 26

8 Massless particles and the chiral kinetic theory 28

9 Spin density matrix and spin polarization vector $\quad 30$

10 Summary $\quad 32$

A Spinors and group theory 33

$\begin{array}{lll}\text { A.1 Massless spinor product } & 36\end{array}$

$\begin{array}{ll}\text { B Massless fermions and Unruh effect } & 37\end{array}$

$\begin{array}{lll}\text { B.1 Proof of the equation (B.4) } & 39\end{array}$

$\begin{array}{lll}\text { C Analytic distillation for pure rotation } & 40\end{array}$

D Analytic distillation for rotation and acceleration $\quad 42$

\section{Introduction}

In a previous paper [1], we presented a new method to calculate exact expressions of mean values involving the free scalar field in the most general global thermodynamic equilibrium in Minkowski space-time, including acceleration and rotation. In this paper, we extend that method to a free quantum field of any spin and particularly the free Dirac field of 
spin $1 / 2$ particles. We obtain exact expressions of several physical quantities, including the covariant Wigner function, which is of great relevance for the relativistic kinetic theory of massive fermions [2-8] as well as the spin density matrix and the spin polarization vector, which are of special interest for the phenomenology of relativistic heavy ion collisions [9]. We demonstrate the viability of our method by comparing the newly found results with those known in the literature for the two special cases of equilibrium with pure acceleration and pure rotation and with those known at the leading perturbative order in thermal vorticity. On top of that, we compute the exact stress energy tensor and axial current for a massless field at equilibrium with both acceleration and rotation. Furthermore, we derive the exact expression of the massless fermions distribution function at global equilibrium, which is very relevant for the chiral kinetic theory [10-21] as well as the exact formula of the spin polarization vector for massive particles as a formal series which extends the known expression at first order.

The density operator describing the most general thermodynamic equilibrium in flat space-time reads [22]:

$$
\widehat{\rho}=\frac{1}{Z} \exp \left[-b_{\mu} \widehat{P}^{\mu}+\frac{1}{2} \varpi_{\mu \nu} \widehat{J}^{\mu \nu}+\zeta \widehat{Q}\right]
$$

where $\widehat{P}^{\mu}$ and $\widehat{J}^{\mu \nu}$ are the generators of translation and Lorentz transformations and $\widehat{Q}$ is a conserved charge; the four-vector $b$ is constant and time-like, $\varpi$ is an anti-symmetric constant tensor, the thermal vorticity, and $\zeta$ is the ratio between the chemical potential and the temperature, i.e. $\zeta=\mu / T$. This operator and its characteristics have been studied in detail elsewhere [23-26]. We just remind that the local temperature $T$ measured by a comoving thermometer is $1 / \sqrt{\beta^{2}}$, where $\beta$ is the four-temperature vector. For the density operator (1.1) $\beta$ is the Killing vector:

$$
\beta_{\mu}=b_{\mu}+\varpi_{\mu \nu} x^{\nu}
$$

and its direction defines the flow velocity $u$ in space-time [25]. The thermal vorticity tensor can be decomposed as:

$$
\varpi_{\mu \nu}=\epsilon_{\mu \nu \rho \sigma} w^{\rho} u^{\sigma}+\left(\alpha_{\mu} u_{\nu}-\alpha_{\nu} u_{\mu}\right)
$$

with:

$$
\alpha^{\mu}=\varpi^{\mu \nu} u_{\nu} \quad w^{\mu}=-\frac{1}{2} \epsilon^{\mu \nu \rho \sigma} \varpi_{\nu \rho} u_{\sigma}
$$

and, at the global equilibrium defined by the equation (1.2), $w$ and $\alpha$ are related to the acceleration and kinematic vorticity fields $A^{\mu}$ and $\omega^{\mu}$, by the simple relations:

$$
\alpha^{\mu}=\frac{A^{\mu}}{T}, \quad w^{\mu}=\frac{\omega^{\mu}}{T} .
$$

It is important to stress that, even though the above definitions evoke a hydrodynamic language, our calculations do not require the system to be a proper fluid (and a system of free particles is certainly not). Indeed, we deal with a global thermodynamic equilibrium situation which - in the form of the density operator (1.1), applies to any system, whether 
very weakly or very strongly interacting. Particularly, our calculation for a free Dirac field means that we seek the equilibrium thermodynamic distributions of a gas of quasi-free fermions where the interaction energy - which would be necessary to achieve equilibrium dynamically - is neglected, just like for the familiar Bose-Einstein or Fermi-Dirac distribution functions. Its relevance for phenomenological situations is further addressed in section 10 .

In ref. [1] we put forward a method to calculate the exact expression of expectation values with the density operator (1.1) (henceforth denoted as thermal expectation values or TEV), based on the factorization of the exponentials in the density operator (1.1) and their analytical continuation to complex thermal vorticity. We introduced a mathematical prescription, dubbed as analytic distillation, to remove the unphysical, non-analytic terms, which makes it possible to obtain the actual physical TEVs by continuing back to real thermal vorticity. In this work, we shall extend this method to the fermionic case.

The paper is organized as follows. In section 2 we review the spinor formalism for the Dirac field used throughout the paper; in section 3 we review the definition and properties of the Wigner function for the Dirac field; in section 4 we derive the exact form of the TEVs of quadratic combinations of creation and annihilation operators for a particle with arbitrary spin $S$. Then, we obtain the exact form of the Wigner function of a free Dirac field and, in section 5, starting from the Wigner function, we derive the exact form of the TEVs of the stress-energy tensor, the vector current and the axial current. In section 6 we introduce and discuss the analytic distillation procedure for the fermionic case and in section 7 we present the results for the two cases of pure acceleration and pure rotation for the massless free Dirac field. In section 8 we derive the exact form of the distribution function for a massless fermions and discuss its relevance for chiral kinetic theory. Finally, in section 9 we derive the exact global equilibrium formula of the spin density matrix and the spin polarization vector.

Notation. In this paper we use the natural units, with $\hbar=c=K=1$. The Minkowskian metric tensor $g$ is $\operatorname{diag}(1,-1,-1,-1)$; for the Levi-Civita symbol we use the convention $\epsilon^{0123}=1$.

We will use the relativistic notation with repeated indices assumed to be saturated. Operators in Hilbert space will be denoted by a wide upper hat, e.g. $\widehat{H}$, except the Dirac field operator which is denoted by a $\Psi$. The symbol $\operatorname{Tr}$ will denote the trace over the Hilbert space of quantum field states, while tr over a finite dimensional vector space.

\section{Basics of the free Dirac field}

Before we get into the main topic of this work, it is necessary to introduce the basic formalism of the Dirac and spin 1/2 particles theory. We adopt a group theoretical framework, which is very convenient for our approach. More details about the formalism can be found in the appendix $\mathrm{A}$. 
It is known that the free Dirac field can be expanded in plane waves:

$$
\Psi(x)=\frac{1}{(2 \pi)^{3 / 2}} \sum_{s=-1 / 2}^{1 / 2} \int \frac{\mathrm{d}^{3} \mathrm{p}}{2 \varepsilon}\left[\widehat{a}_{s}(p) u_{s}(p) \mathrm{e}^{-i p \cdot x}+\widehat{b}_{s}^{\dagger}(p) v_{s}(p) \mathrm{e}^{i p \cdot x}\right],
$$

where $\widehat{a}_{s}(p)$ and $\widehat{b}_{s}^{\dagger}(p)$ are the annihilation and creation operators for a particle and antiparticle of polarization state $s$ (either a spin component or helicity) and momentum $p ; \varepsilon$ is the energy $\sqrt{\mathbf{p}^{2}+m^{2}}$. In the $(2.1)$, the covariant anti-commutation rules are implied:

$$
\begin{aligned}
& \left\{\widehat{a}_{s}(p), \widehat{a}_{t}^{\dagger}\left(p^{\prime}\right)\right\}=2 \varepsilon \delta_{s t} \delta^{3}\left(\mathbf{p}-\mathbf{p}^{\prime}\right), \\
& \left\{\widehat{b}_{s}(p), \widehat{b}_{t}^{\dagger}\left(p^{\prime}\right)\right\}=2 \varepsilon \delta_{s t} \delta^{3}\left(\mathbf{p}-\mathbf{p}^{\prime}\right) .
\end{aligned}
$$

The spinors $u_{s}(p)$ and $v_{s}(p)$ are column vectors with four components. To make derivations more compact, it is convenient to introduce $4 \times 2$ matrices $\mathrm{U}(p)$ and $V(p)$ for the spinors, as well as arranging the creation and destruction operators in column vectors:

$$
\mathrm{U}(p)=\left(u_{1 / 2}(p), u_{-1 / 2}(p)\right) \quad \widehat{A}(p)=\left(\begin{array}{c}
\widehat{a}_{1 / 2}(p) \\
\widehat{a}_{-1 / 2}(p)
\end{array}\right)
$$

and similarly for $V(p)$ and $\widehat{B}^{\dagger}(p)$. With the notation (2.2), the field expansion can be rewritten as:

$$
\Psi(x)=\frac{1}{(2 \pi)^{3 / 2}} \int \frac{\mathrm{d}^{3} \mathrm{p}}{2 \varepsilon}\left[\mathrm{U}(p) \widehat{A}(p) \mathrm{e}^{-i p \cdot x}+V(p) \widehat{B}^{\dagger}(p) \mathrm{e}^{i p \cdot x}\right]
$$

with $\mathrm{U}(p) \widehat{A}(p)$ which is now a multiplication of a matrix $4 \times 2$ by a $2 \times 1$.

The spinors $\mathrm{U}(p)$ and $V(p)$ are related to the so-called standard Lorentz transformation, henceforth denoted by $[p]$, that transforms a chosen standard four-vector $\mathfrak{p}$ into the fourmomentum $p$. The spinor form is dictated by the request for the field $\Psi(x)$ to transform according to the irreducible representation $(0,1 / 2) \oplus(1 / 2,0)$ of the orthochronous Lorentz group $\mathrm{SO}(1,3)^{\uparrow}[27]$. Therefore, they can be obtained through the application of the representation of the standard Lorentz transformation to the spinor $U(\mathfrak{p})$ associated to the standard four-vector $\mathfrak{p}$ (see appendix A):

$$
\mathrm{U}(p)=\left(\begin{array}{cc}
D([p]) & 0 \\
0 & D([p])^{\dagger-1}
\end{array}\right) \mathrm{U}(\mathfrak{p}), \quad V(p)=\left(\begin{array}{cc}
D([p]) & 0 \\
0 & D([p])^{\dagger-1}
\end{array}\right) V(\mathfrak{p}),
$$

where $D\left(\right.$ ) stands for the 2-dimensional $(0,1 / 2)$ representation $D^{(0,1 / 2)}$ of $\mathrm{SO}(1,3)^{\uparrow}$. In general, the matrix:

$$
S(\Lambda)=\left(\begin{array}{cc}
D(\Lambda) & 0 \\
0 & D(\Lambda)^{\dagger-1}
\end{array}\right)
$$

is the representation of a Lorentz transformation in the $(0,1 / 2) \oplus(1 / 2,0)$ full representation of the Lorentz group including reflections. The generators of $\mathrm{SO}(1,3)^{\uparrow}$ in this representations are given by:

$$
\left(\begin{array}{cc}
D^{(0,1 / 2)}\left(J^{\mu \nu}\right) & 0 \\
0 & D^{(1 / 2,0)}\left(J^{\mu \nu}\right)
\end{array}\right)=\frac{i}{4}\left[\gamma^{\mu}, \gamma^{\nu}\right] \equiv \Sigma^{\mu \nu}
$$


so that:

$$
S(\Lambda)=\exp \left[-i \frac{\phi^{\mu \nu}}{2} \Sigma_{\mu \nu}\right]
$$

and where the $\gamma$ matrices are those in the so-called Weyl representation:

$$
\gamma^{\mu}=\left(\begin{array}{cc}
0 & \sigma^{\mu} \\
\bar{\sigma}^{\mu} & 0
\end{array}\right), \quad \gamma_{5}=\left(\begin{array}{cc}
I & 0 \\
0 & -I
\end{array}\right),
$$

with $\sigma_{\mu}=\left(I, \sigma_{1}, \sigma_{2}, \sigma_{3}\right), \sigma_{i}$ being the Pauli matrices, $\bar{\sigma}_{\mu}=\left(I,-\sigma_{1},-\sigma_{2},-\sigma_{3}\right)$ and $\sigma^{\mu}=$ $\eta^{\mu \nu} \sigma_{\nu}$. The spinors $\mathrm{U}(\mathfrak{p}), V(\mathfrak{p})$ in eq. (2.4) read:

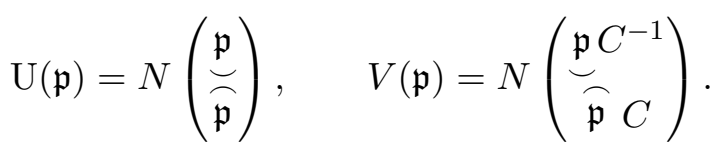

In the (2.8), $N$ is a normalization factor and $C=i \sigma_{2}$; the lower arc stands for the hermitian matrix corresponding to a four-vector:

$$
X \equiv X^{\mu} \sigma_{\mu}
$$

where the upper arc stands for the hermitian matrix corresponding to the reflected vector:

$$
\overparen{X}=X^{\mu} \bar{\sigma}_{\mu}
$$

such definitions are well known in the construction of the $\mathrm{SL}(2, \mathbb{C})-\mathrm{SO}(1,3)^{\uparrow}$ morphism [28]. The appearance of the matrix $C$ in the $V$ spinor is dictated by the required transformation properties of the $\widehat{A}$ and $\widehat{B}$ operators under charge conjugation.

The standard vector $\mathfrak{p}$ and the normalization factor $N$ depend on whether $m \neq 0$ or $m=0$. In the massive case, $\mathfrak{p}^{\mu}=(m, \mathbf{0}), N=1 / \sqrt{m}$ and the spinor $\mathrm{U}(\mathfrak{p})$ is then:

$$
\mathrm{U}(\mathfrak{p})=\sqrt{m}\left(\begin{array}{l}
I \\
I
\end{array}\right)
$$

with $I$ the $2 \times 2$ identity matrix. On the other hand, in the massless case, the standard vector it is usually chosen to be $\mathfrak{p}^{\mu}=(\kappa, 0,0, \kappa)$, where $\kappa$ is some fixed energy value. The normalization factor is $1 / \sqrt{2 \kappa}$ and the spinor $U(\mathfrak{p})$ reads:

$$
\mathrm{U}(\mathfrak{p})=\frac{1}{\sqrt{2 \kappa}}\left(\begin{array}{cc}
2 \kappa & 0 \\
0 & 0 \\
0 & 0 \\
0 & 2 \kappa
\end{array}\right)=\sqrt{2 \kappa}\left(\begin{array}{ll}
1 & 0 \\
0 & 0 \\
0 & 0 \\
0 & 1
\end{array}\right)
$$

Because of the above form, it turns out the column spinor with helicity $+1 / 2$, that is $u_{+}(p)$ has only the upper two components non-vanishing and it is the first column of the matrix $\mathrm{U}(p)$. Conversely, the spinor $u_{-}(p)$ with helicity $-1 / 2$ is the second column of the $\mathrm{U}(p)$ and has the upper two-component vanishing.

It should be stressed that the spinors depend, as it is apparent from their definition (2.2), on both the chosen standard Lorentz transformation $[p]$ and the standard vector 
p. Nevertheless, also the particle states [29] — that is the creation and annihilation operators - depend on the particular choice of $[p]$ and $\mathfrak{p}$, so as to make the combination $\mathrm{U}(p) \widehat{A}(p)$ and $V(p) \widehat{B}^{\dagger}(p)$, hence the field (2.3), independent thereof.

The spinors $\mathrm{U}(p)$ and $V(p)$, however constructed, fulfill the Dirac equation in momentum space, i.e. with $\not p=\gamma^{\mu} p_{\mu}$ :

$$
(\not p-m) U(p)=0, \quad(\not p+m) V(p)=0 .
$$

what can be proved by taking into account the $\mathrm{SL}(2, \mathbb{C})-\mathrm{SO}(1,3)^{\uparrow}$ correspondence (see appendix A for the proof). Note that, from (2.7) and (2.9), (2.10):

$$
\not p=\left(\begin{array}{cc}
0 & p \\
\widehat{p} & 0
\end{array}\right) .
$$

It is possible to write the spinors in a convenient fashion by picking a particular standard Lorentz transformation $[p]$. For the massive case, with $\mathfrak{p}^{\mu}=(m, \mathbf{0})$ and $[p]$ the pure boost taking $\mathfrak{p}$ to $p$, one has (see appendix $\mathrm{A}$ ):

$$
U(p)=\frac{1}{\sqrt{2(\varepsilon+m)}}(\not p+m)\left(\begin{array}{l}
I \\
I
\end{array}\right)
$$

While for the massless case, by choosing $\mathfrak{p}^{\mu}=(\kappa, 0,0, \kappa)$ and $[p]$ the composition of a Lorentz boost along the $z$ axis and a rotation taking $\hat{\mathbf{k}}$ into $\hat{\mathbf{p}}$ with axis $\hat{\mathbf{k}} \times \hat{\mathbf{p}}$ (see appendix A):

$$
\mathrm{U}(p)=\frac{1}{\sqrt{2 p \cdot \overline{\mathfrak{p}}}} \not p \gamma^{0} \mathrm{U}(\mathfrak{p}),
$$

where the bar over a four-vector implies the reflection of its space components, that is $\bar{X}^{\mu}=\left(X^{0},-\mathbf{X}\right)$. With this compact form, it can be readily checked that the spinors fulfill the following relations:

$$
\begin{array}{rlrl}
\overline{\mathrm{U}}(p) \mathrm{U}(p) & =2 m I & \mathrm{U}(p) \overline{\mathrm{U}}(p) & =(\not p+m) \\
\bar{V}(p) V(p) & =-2 m I & V(p) \bar{V}(p) & =(\not p-m)
\end{array}
$$

in both the massive and the massless case.

It is also convenient to define a matrix $\mathcal{C}=i \gamma^{2}$ which is very useful to work out most of the expressions involving the anti-particle spinors $V(p)$ for, from the (2.8) and the definitions of the $\gamma^{\prime}$ 's in (2.7):

$$
V(p)=\mathcal{C U}(p)^{*}
$$

This matrix is involved in two more useful relations:

$$
\gamma^{0} \mathcal{C} S(\Lambda)^{T} \mathcal{C} \gamma^{0}=S(\Lambda)^{-1}, \quad \quad \gamma^{0} \mathcal{C} \gamma^{\mu T} \mathcal{C} \gamma^{0}=-\gamma^{\mu}
$$




\section{The covariant Wigner function of the free Dirac field}

All relevant expectation values in statistical quantum field theory can be obtained from the covariant Wigner function. For the free Dirac field, this is defined as [30]:

$$
W_{A B}(x, k)=-\frac{1}{(2 \pi)^{4}} \int \mathrm{d}^{4} y \mathrm{e}^{-i k \cdot y} \operatorname{Tr}\left(\widehat{\rho}: \Psi_{A}\left(x-\frac{y}{2}\right) \bar{\Psi}_{B}\left(x+\frac{y}{2}\right):\right),
$$

where the colons imply normal ordering, $\bar{\Psi}$ denotes the Dirac conjugate field $\bar{\Psi}=\Psi^{\dagger} \gamma^{0}$, and $A, B$ are the spinorial indices, running from 1 to 4 . The Wigner function (3.1) is a $4 \times 4$ matrix fulfilling:

$$
W^{\dagger}(x, k)=\gamma^{0} W(x, k) \gamma^{0}
$$

The argument $k$ is a general four-vector and it is not on-shell, even in the free field case. Indeed, by plugging (2.1) into (3.1), one obtains: ${ }^{1}$

$$
\begin{aligned}
W(x, k)= & \frac{1}{(2 \pi)^{3}} \sum_{s, t} \int \frac{\mathrm{d}^{3} \mathrm{p}}{2 \varepsilon} \frac{\mathrm{d}^{3} \mathrm{p}^{\prime}}{2 \varepsilon^{\prime}}\left\{\mathrm { e } ^ { i x \cdot ( p ^ { \prime } - p ) } \left[\left\langle\widehat{a}_{t}^{\dagger}\left(p^{\prime}\right) \widehat{a}_{s}(p)\right\rangle u_{s}(p) \bar{u}_{t}\left(p^{\prime}\right) \delta^{4}\left(k-\frac{p+p^{\prime}}{2}\right)\right.\right. \\
& \left.-\left\langle\widehat{b}_{s}^{\dagger}\left(p^{\prime}\right) \widehat{b}_{t}(p)\right\rangle v_{s}\left(p^{\prime}\right) \bar{v}_{t}(p) \delta^{4}\left(k+\frac{p+p^{\prime}}{2}\right)\right]-\left[\mathrm{e}^{i\left(p+p^{\prime}\right) \cdot x}\left\langle\widehat{b}_{t}^{\dagger}\left(p^{\prime}\right) \widehat{a}_{s}^{\dagger}(p)\right\rangle v_{t}\left(p^{\prime}\right) \bar{u}_{s}(p)\right. \\
& \left.\left.+\mathrm{e}^{-i\left(p+p^{\prime}\right) \cdot x}\left\langle\widehat{a}_{t}(p) \widehat{b}_{s}\left(p^{\prime}\right)\right\rangle u_{t}(p) \bar{v}_{s}\left(p^{\prime}\right)\right] \delta^{4}\left(k-\frac{p-p^{\prime}}{2}\right)\right\}
\end{aligned}
$$

The expansion (3.2) makes it apparent that the Wigner function of a free field can be decomposed into particle, antiparticle and space-like terms:

$$
W(x, k)=\theta\left(k^{2}\right) \theta\left(k^{0}\right) W_{+}(x, k)+\theta\left(k^{2}\right) \theta\left(-k_{0}\right) W_{-}(x, k)+\theta\left(-k^{2}\right) W_{S}(x, k),
$$

which can be singled out multiplying the Wigner function $W(x, k)$ by appropriate Heaviside $\theta$ functions.

Using the Dirac equation and integrating by parts, one can show that $W$ is a solution of a partial differential equation, known as Wigner equation:

$$
\left(\frac{i}{2} \not \partial+\not k-m\right) W(x, k)=0 .
$$

It should be stressed that the Wigner equation is independent of the statistical operator, meaning that the equation provides no information about the thermodynamic equilibrium value. The Wigner equation is, in this respect, only a constraint that the physical $W(x, k)$ must fulfill. Thus far, the only known non-trivial solution of this equation is the one associated with global homogeneous equilibrium, at constant four-temperature. The form derived in this work, the Wigner function at global thermodynamic equilibrium with acceleration and rotation is a new, non-trivial, solution of the (3.4).

The most important feature of the Wigner function is that it allows to express the expectation values of local operators as integrals in $k$. For instance, the mean values of the

\footnotetext{
${ }^{1}$ Henceforth, $\langle\widehat{X}\rangle$ is a shorthand for $\operatorname{Tr}(\widehat{\rho} \widehat{X})$.
} 
vector current, axial current, and canonical stress-energy tensor of the free Dirac field can be written as:

$$
\begin{aligned}
j^{\mu}(x) & \equiv\left\langle: \widehat{j}^{\mu}(x):\right\rangle=\left\langle: \bar{\Psi}(x) \gamma^{\mu} \Psi(x):\right\rangle=\operatorname{tr}\left(\gamma^{\mu} \int \mathrm{d}^{4} k W(x, k)\right), \\
j_{A}^{\mu}(x) & \equiv\left\langle: \widehat{j}_{\mathrm{A}}^{\mu}(x):\right\rangle=\left\langle: \bar{\Psi}(x) \gamma^{\mu} \gamma_{5} \Psi(x):\right\rangle=\operatorname{tr}\left(\gamma^{\mu} \gamma_{5} \int \mathrm{d}^{4} k W(x, k)\right), \\
T_{C}^{\mu \nu}(x) & \equiv\left\langle: \widehat{T}_{C}^{\mu \nu}(x):\right\rangle=\left\langle: \frac{i}{2}\left(\bar{\Psi}(x) \gamma^{\mu} \stackrel{\leftrightarrow}{\partial^{\nu}} \Psi(x):\right\rangle=\operatorname{tr}\left(\gamma^{\mu} \int \mathrm{d}^{4} k k^{\nu} W(x, k)\right),\right.
\end{aligned}
$$

where $\overleftrightarrow{\partial^{\nu}}=\overrightarrow{\partial^{\nu}}-\overleftarrow{\partial^{\nu}}$

Such expressions are very useful, but since the pseudo-momentum variable $k$ is offshell, as we have seen, it is desirable, in several circumstances, to deal with a distribution function with only on-shell momentum as argument, like in the classical kinetic theory. To define it starting from the Wigner function, one could follow the method used in the scalar field case [1], namely recast the mean current (3.5a) as an integral over on-shell momenta multiplied by a four-vector $p^{\mu}$. This method can be applied to particles and antiparticles separately, by taking advantage of the decomposition (3.3); one can then focus on the particle contribution only, the antiparticle being easily obtained from it. By using the (3.2) into the definition (3.5a), we can then write the particle contribution to the mean current as:

$$
j_{+}^{\mu}(x)=\frac{1}{(2 \pi)^{3}} \sum_{s, t} \int \frac{\mathrm{d}^{3} \mathrm{p}}{2 \varepsilon} \frac{\mathrm{d}^{3} \mathrm{p}^{\prime}}{2 \varepsilon^{\prime}} \mathrm{e}^{i\left(p^{\prime}-p\right) \cdot x}\left\langle\widehat{a}_{t}^{\dagger}\left(p^{\prime}\right) \widehat{a}_{s}(p)\right\rangle \bar{u}_{t}\left(p^{\prime}\right) \gamma^{\mu} u_{s}(p) .
$$

The next step would be to write the above current in terms of a distribution function $f(x, p)$ with on-shell momenta. In general, the current in (3.6) can be written as a momentum integral of a "phase-space" vector field depending on both momentum and space-time point:

$$
j_{+}^{\mu}(x)=\int \frac{\mathrm{d}^{3} \mathrm{p}}{\varepsilon} \mathcal{J}^{\mu}(x, p) .
$$

Nevertheless, unlike in a classical theory, in general $\mathcal{J}$ is not directed along $p$. Hence, in order to identify a suitable distribution function $f(x, p)$, one has to decompose the phase space vector field into the sum of a term parallel to $p^{\mu}$ and terms orthogonal to it:

$$
\mathcal{J}^{\mu}(x, p)=p^{\mu} f(x, p)+N^{\mu}(x, p), \quad N \cdot p=0 .
$$

For the massive Dirac field, one can obtain such decomposition simply by applying the Gordon identity. On the other hand, the massless case presents some interesting complications that are dealt with in section 8 in order to compare with some recent achievements in chiral kinetic theory.

\section{Exact Wigner function at global thermodynamic equilibrium}

In this section, an exact form of the Wigner function (3.1) for the general global thermodynamic equilibrium density operator (1.1) will be derived. It will be obtained with the 
same method used in ref. [1]: a factorization of the density operator and an iterative procedure to calculate the expectation values $\left\langle\widehat{a}_{s}^{\dagger}(p) \widehat{a}_{t}\left(p^{\prime}\right)\right\rangle$ with imaginary thermal vorticity. The integrals of the Wigner function (3.5) are then analytically continued to real thermal vorticity to obtain the physical values. For the sake of simplicity, we set $\zeta=0$ in the density operator (1.1); the extension to a finite $\zeta$ is straightforward.

\subsection{Expectation values}

As has been mentioned, the method presented in [1] involves two steps. The first step is the factorization of the density operator (1.1):

$$
\widehat{\rho}=\frac{1}{Z} \exp \left[-b_{\mu} \widehat{P}^{\mu}+\frac{\varpi_{\mu \nu}}{2} \widehat{J}^{\mu \nu}\right]=\frac{1}{Z} \exp \left[-\widetilde{b}_{\mu}(\varpi) \widehat{P}^{\mu}\right] \exp \left[\frac{\varpi_{\mu \nu}}{2} \widehat{J}^{\mu \nu}\right],
$$

where the tilde transform of a vector is defined as:

$$
\widetilde{b}^{\mu}(\varpi)=\sum_{k=0}^{\infty} \frac{i^{k}}{(k+1) !}(\underbrace{\varpi_{\alpha_{1}}^{\mu} \varpi_{\alpha_{2}}^{\alpha_{1}} \ldots \varpi_{\alpha_{k}}^{\alpha_{k-1}}}_{k \text { times }}) b^{\alpha_{k}} .
$$

To obtain a finite expression of $\left\langle\widehat{a}_{s}^{\dagger}(p) \widehat{a}_{t}\left(p^{\prime}\right)\right\rangle$ it is necessary to deal with an imaginary thermal vorticity, setting $\varpi_{\mu \nu}=-i \phi_{\mu \nu}$. Thereby, one can deal with an actual Lorentz transformation $\widehat{\Lambda}=\exp [-i \phi: \widehat{J} / 2]$ and the density operator (4.1) becomes a product of an exponential of the four-momentum times the unitary representation of a Lorentz transformation in the Hilbert space:

$$
\widehat{\rho}=\frac{1}{Z} \exp \left[-\widetilde{b}_{\mu}(-i \phi) \widehat{P}^{\mu}\right] \exp \left[-i \frac{\phi_{\mu \nu}}{2} \widehat{J}^{\mu \nu}\right]=\frac{1}{Z} \exp \left[-\widetilde{b}_{\mu}(-i \phi) \widehat{P}^{\mu}\right] \widehat{\Lambda} .
$$

This form makes it possible to use Poincaré group representation theory to calculate $\left\langle\widehat{a}_{s}^{\dagger}(p) \widehat{a}_{t}\left(p^{\prime}\right)\right\rangle$. Let $S$ be the spin of a particle (or helicity, for massless particles) and $s$ its polarization state label running from $-S$ to $S$ ( $\pm S$ for massless particles). A creation operator transforms under $\widehat{\Lambda}$ as:

$$
\widehat{\Lambda} \widehat{a}_{s}^{\dagger}(p) \widehat{\Lambda}^{\dagger}=\sum_{r} D^{S}(W(\Lambda, p))_{r s} \widehat{a}_{r}^{\dagger}(\Lambda p)
$$

where $W(\Lambda, p)=[\Lambda p]^{-1} \Lambda[p]$ is a member of the so-called little group, that is the subgroup of Lorentz transformations leaving the standard momentum $\mathfrak{p}$ invariant; $D^{S}$ stands for the $(0, S)$-th finite-dimensional representation of the Lorentz group (see appendix A). As we have seen, for massive particles $\mathfrak{p}=(m, \mathbf{0})$ and the little group is just $\mathrm{SO}(3)$; in this case, the $W(\Lambda, p)$ matrix is the so-called Wigner rotation. For massless particles, the little group is the euclidean group (rotations and translations) in two dimensions, the $\operatorname{ISO}(2)$ group; yet, the translations do not play a physical role and the transformation $D(W(\Lambda, p))$ appearing in (4.3) boils down to a phase: $D(W(\Lambda, p))_{r s}=\mathrm{e}^{-i r \theta(\Lambda, p)} \delta_{r s}[27]$. 
The second step to calculate $\left\langle\widehat{a}_{s}^{\dagger}(p) \widehat{a}_{t}(p)\right\rangle$ involves the use of commutation or anticommutation relations and the transformation rule (4.3):

$$
\begin{aligned}
\left\langle\widehat{a}_{s}^{\dagger}(p) \widehat{a}_{t}\left(p^{\prime}\right)\right\rangle= & \frac{1}{Z} \operatorname{Tr}\left(\mathrm{e}^{-\widetilde{b} \cdot \widehat{P}} \widehat{\Lambda}_{a_{s}^{\dagger}}^{\dagger}(p) \widehat{a}_{t}\left(p^{\prime}\right)\right) \\
= & \frac{1}{Z} \sum_{r} D^{S}(W(\Lambda, p))_{r s} \operatorname{Tr}\left(\mathrm{e}^{-\widetilde{b} \cdot \widehat{P}} \widehat{a}_{r}^{\dagger}(\Lambda p) \widehat{\Lambda} \widehat{a}_{t}\left(p^{\prime}\right)\right) \\
= & \sum_{r} D^{S}(W(\Lambda, p))_{r s} \mathrm{e}^{-\widetilde{b} \cdot \Lambda p}\left\langle\widehat{a}_{t}\left(p^{\prime}\right) \widehat{a}_{r}^{\dagger}(\Lambda p)\right\rangle \\
= & (-1)^{2 S} \sum_{r} D^{S}(W(\Lambda, p))_{r s} \mathrm{e}^{-\widetilde{b} \cdot \Lambda p}\left\langle\widehat{a}_{r}^{\dagger}(\Lambda p) \widehat{a}_{t}\left(p^{\prime}\right)\right\rangle \\
& +2 \varepsilon \mathrm{e}^{-\widetilde{b} \cdot \Lambda p} D^{S}(W(\Lambda, p))_{t s} \delta^{3}\left(\Lambda \boldsymbol{p}-\boldsymbol{p}^{\prime}\right),
\end{aligned}
$$

where the factor $(-1)^{2 S}$ selects commutation or anticommutation for integer or half-integer spin respectively. A special solution of the equation (4.4) can be found by means of the iterative method described in ref. [1]. One starts by taking the rightmost term in the (4.4) as the leading order approximation of the expectation value:

$$
\left\langle\widehat{a}_{s}^{\dagger}(p) \widehat{a}_{t}\left(p^{\prime}\right)\right\rangle \sim 2 \varepsilon \mathrm{e}^{-\widetilde{b} \cdot \Lambda p} D^{S}(W(\Lambda, p))_{t s} \delta^{3}\left(\Lambda \boldsymbol{p}-\boldsymbol{p}^{\prime}\right) .
$$

Then, the leading order solution is fed into the right hand side of the (4.4) to get an updated expression of the solution, which is the sum of two terms involving delta-functions:

$$
\begin{aligned}
\left\langle\widehat{a}_{s}^{\dagger}(p) \widehat{a}_{t}\left(p^{\prime}\right)\right\rangle \sim & 2 \varepsilon(-1)^{2 S} \sum_{r} D^{S}(W(\Lambda, p))_{r s} D^{S}(W(\Lambda, \Lambda p))_{t r} \mathrm{e}^{-\widetilde{b} \cdot\left(\Lambda p+\Lambda^{2} p\right)} \delta^{3}\left(\Lambda^{2} \boldsymbol{p}-\boldsymbol{p}^{\prime}\right) \\
& +2 \varepsilon \mathrm{e}^{-\widetilde{b} \cdot \Lambda p} D^{S}(W(\Lambda, p))_{t s} \delta^{3}\left(\Lambda \boldsymbol{p}-\boldsymbol{p}^{\prime}\right) .
\end{aligned}
$$

The product of two transformations of the little group can be written in a more compact form:

$$
D^{S}(W(\Lambda, \Lambda p)) D^{S}(W(\Lambda, p))=D^{S}\left([\Lambda \Lambda p]^{-1} \Lambda[\Lambda p][\Lambda p]^{-1} \Lambda[p]\right)=D^{S}\left(W\left(\Lambda^{2}, p\right)\right),
$$

and the updated solution becomes:

$$
\begin{aligned}
\left\langle\widehat{a}_{s}^{\dagger}(p) \widehat{a}_{t}\left(p^{\prime}\right)\right\rangle \sim & 2 \varepsilon(-1)^{2 S} D^{S}\left(W\left(\Lambda^{2}, p\right)\right)_{t s} \mathrm{e}^{-\widetilde{b} \cdot\left(\Lambda p+\Lambda^{2} p\right)} \delta^{3}\left(\Lambda^{2} \boldsymbol{p}-\boldsymbol{p}^{\prime}\right) \\
& +2 \varepsilon \mathrm{e}^{-\widetilde{b} \cdot \Lambda p} D^{S}(W(\Lambda, p))_{t s} \delta^{3}\left(\Lambda \boldsymbol{p}-\boldsymbol{p}^{\prime}\right) .
\end{aligned}
$$

Iterating, one eventually obtains a series of delta functions:

$$
\left\langle\widehat{a}_{s}^{\dagger}(p) \widehat{a}_{t}\left(p^{\prime}\right)\right\rangle=2 \varepsilon^{\prime} \sum_{n=1}^{\infty}(-1)^{2 S(n+1)} \delta^{3}\left(\Lambda^{n} \boldsymbol{p}-\boldsymbol{p}^{\prime}\right) D^{S}\left(W\left(\Lambda^{n}, p\right)\right)_{t s} \mathrm{e}^{-\widetilde{b} \cdot \sum_{k=1}^{n} \Lambda^{k} p} .
$$

A similar formula can be obtained for the anti-particle quadratic combination $\left\langle\widehat{b}_{s}^{\dagger}(p) \widehat{b}_{t}\left(p^{\prime}\right)\right\rangle$. As also mentioned in [1], all other expectation values of combinations of creation and annihilation operators, like e.g. $\widehat{a}_{s}^{\dagger}(p) \widehat{a}_{t}^{\dagger}\left(p^{\prime}\right), \widehat{a}_{s}(p) a_{t}\left(p^{\prime}\right)$ and mixed particle-antiparticle combinations, vanish because the global equilibrium density operator (1.1), depending 
on conserved generators of symmetry transformations, cannot change the number of particles/antiparticles in a given state. This can be confirmed by an explicit iterative calculation.

In the trivial case of vanishing thermal vorticity $\varpi=0$, which implies $\Lambda=I$, it is straightforward to sum the right hand side of the eq. (4.5):

$$
\left\langle\widehat{a}_{s}^{\dagger}(p) \widehat{a}_{t}\left(p^{\prime}\right)\right\rangle=2 \varepsilon^{\prime} \sum_{n=1}^{\infty}(-1)^{2 S(n+1)} \delta^{3}\left(\boldsymbol{p}-\boldsymbol{p}^{\prime}\right) \delta_{t s} \mathrm{e}^{-n b \cdot p}=2 \varepsilon \delta^{3}\left(\boldsymbol{p}-\boldsymbol{p}^{\prime}\right) \delta_{t s} \frac{1}{\mathrm{e}^{b \cdot p}+(-1)^{2 S+1}}
$$

reproducing the familiar Bose-Einstein and the Fermi-Dirac distributions. This makes it clear that the series in $n$ corresponds to the typical expansion of the quantum statistics and that the $n>1$ terms are the corrections are the Boltzmann statistics term, which is the first, with $n=1$.

As has been mentioned, the solution (4.5) becomes the physical mean value of the number operator at general global equilibrium only after the analytic continuation to real thermal vorticity. However, this continuation cannot be done at the level of (4.5), because of the singular delta functions. Nonetheless, the (4.5) can be used once momentum integration is carried out.

\subsection{The Wigner function}

We are now in a position to calculate the Wigner function at global equilibrium, for imaginary thermal vorticity, both for massive and massless particles. Feeding the (4.5) into the (3.2) and taking into account the vanishing of the expectation values of some combinations as discussed above, one gets:

$$
\begin{aligned}
W(x, k)= & \frac{1}{(2 \pi)^{3}} \int \frac{\mathrm{d}^{3} \mathrm{p}}{2 \varepsilon} \sum_{n=1}^{\infty}(-1)^{n+1} \mathrm{e}^{-i x \cdot\left(\Lambda^{n} p-p\right)} \mathrm{e}^{-\widetilde{b} \cdot \sum_{k=1}^{n} \Lambda^{k} p} \\
& \times \sum_{r s}\left[u_{r}\left(\Lambda^{n} p\right) D\left(W\left(\Lambda^{n}, p\right)\right)_{r s} \bar{u}_{s}(p) \delta^{4}\left(k-\frac{\Lambda^{n} p+p}{2}\right)\right. \\
& \left.-v_{r}(p) D\left(W\left(\Lambda^{n}, p\right)\right)_{s r} \bar{v}_{s}\left(\Lambda^{n} p\right) \delta^{4}\left(k+\frac{\Lambda^{n} p+p}{2}\right)\right],
\end{aligned}
$$

or, using the compact $4 \times 2$ spinor notation $(2.2)$ :

$$
\begin{aligned}
& W(x, k) \\
& =\frac{1}{(2 \pi)^{3}} \int \frac{\mathrm{d}^{3} \mathrm{p}}{2 \varepsilon} \sum_{n=1}^{\infty}(-1)^{n+1} \mathrm{e}^{-i x \cdot\left(\Lambda^{n} p-p\right)} \mathrm{e}^{-\widetilde{b} \cdot \sum_{k=1}^{n} \Lambda^{k} p} \times \\
& {\left[U\left(\Lambda^{n} p\right) D\left(W\left(\Lambda^{n}, p\right)\right) \bar{U}(p) \delta^{4}\left(k-\frac{\Lambda^{n} p+p}{2}\right)-V(p) D\left(W\left(\Lambda^{n}, p\right)\right)^{T} \bar{V}\left(\Lambda^{n} p\right) \delta^{4}\left(k+\frac{\Lambda^{n} p+p}{2}\right)\right] .}
\end{aligned}
$$

The above expression can be further worked out by using the transformation rules of the spinors under a Lorentz transformation (see appendix A):

$$
\mathrm{U}(\Lambda p) D(W(\Lambda, p))^{\dagger-1}=S(\Lambda) \mathrm{U}(p)
$$


where $S(\Lambda)$ is given by the eq. (2.6), and taking advantage of the unitarity of the $D(W)$ matrices. By using the eq. (2.11) and the basic representation theory rule $S\left(\Lambda_{1} \Lambda_{2}\right)=$ $S\left(\Lambda_{1}\right) S\left(\Lambda_{2}\right)$, we thus have:

$$
S\left(\Lambda^{n}\right)=S(\Lambda)^{n}
$$

and:

$$
U\left(\Lambda^{n} p\right) D\left(W\left(\Lambda^{n}, p\right)\right) \bar{U}(p)=S(\Lambda)^{n} U(p) \bar{U}(p)=S(\Lambda)^{n}(\not p+m) .
$$

Similarly, for antiparticles, keeping in mind that $V^{*}=\mathcal{C} U$ :

$$
\begin{aligned}
V(p) D\left(W\left(\Lambda^{n}, p\right)\right)^{T} \bar{V}\left(\Lambda^{n} p\right) & =-\left(\gamma_{0} \mathcal{C} U\left(\Lambda^{n} p\right) W\left(\Lambda^{n}, p\right) \bar{U}(p) \mathcal{C} \gamma^{0}\right)^{T} \\
& =-\gamma^{0} \mathcal{C}\left(m+\not p^{T}\right) S(\Lambda)^{n T} \mathcal{C} \gamma^{0}=-(m-\not p) S(\Lambda)^{-n}
\end{aligned}
$$

where we have used the relations (2.12). Therefore, the Wigner function can be finally written as:

$$
\begin{aligned}
W(x, k)= & \frac{1}{(2 \pi)^{3}} \int \frac{\mathrm{d}^{3} \mathrm{p}}{2 \varepsilon} \sum_{n=1}^{\infty}(-1)^{n+1} \mathrm{e}^{-i x \cdot\left(\Lambda^{n} p-p\right)} \mathrm{e}^{-\widetilde{b} \cdot \sum_{k=1}^{n} \Lambda^{k} p} \times \\
& {\left[S(\Lambda)^{n}(m+\not p) \delta^{4}\left(k-\frac{\Lambda^{n} p+p}{2}\right)+(m-\not p) S(\Lambda)^{-n} \delta^{4}\left(k+\frac{\Lambda^{n} p+p}{2}\right)\right] . }
\end{aligned}
$$

All the relations used to obtain the above expression hold in the massive and in the massless case as well. Therefore, the Wigner function for a massless Dirac field is simply obtained setting $m=0$ in the eq. (4.8).

It can be shown that the series (4.8) is a non-trivial solution of the Wigner equation (3.4). We will confine ourselves to the particle term, the calculations for the antiparticle one being very similar. The only dependence on the coordinate in the Wigner function is in the exponential, so we can write:

$$
\frac{i}{2} \not \partial \mapsto \frac{1}{2}(\Lambda \not p-\not p)=\Lambda^{p} p-\not k,
$$

where we have taken advantage of the $\delta$ function. Now, since the matrices $p$ and $\widehat{p}$ transform under Lorentz transformation according to

$$
D(\Lambda) \underbrace{p} D(\Lambda)^{\dagger}=\Lambda p, \quad D(\Lambda)^{\dagger-1} \widehat{p} D(\Lambda)^{-1}=\widehat{\Lambda p},
$$

and using $\underbrace{p} \widehat{p}=m^{2}$ (see appendix A), we can work out the matrix product:

$$
\begin{aligned}
\Lambda_{p}^{n p} S(\Lambda)^{n}(m+\not p) & =\left(\begin{array}{cc}
0 & \Lambda^{n} p \\
\widehat{C}^{n} p & 0
\end{array}\right)\left(\begin{array}{cc}
\Lambda^{n} & 0 \\
0 & \Lambda^{n \dagger^{-1}}
\end{array}\right)\left(\begin{array}{cc}
m & p \\
\widehat{p} & m
\end{array}\right) \\
& =\left(\begin{array}{cc}
0 & \Lambda^{n} \underline{p} \\
\Lambda^{n \dagger^{-1}} \widehat{p}
\end{array}\right)\left(\begin{array}{ll}
m & \underline{-} \\
\widehat{p} & m
\end{array}\right) \\
& =\left(\begin{array}{cc}
\Lambda^{n} m^{2} & m \Lambda^{n} \underline{p} \\
m \Lambda^{n \dagger^{-\dagger}} & m^{2} \Lambda^{n \dagger^{-1}}
\end{array}\right) \\
& =m S(\Lambda)^{n}(m+\not p) .
\end{aligned}
$$


whence it ensues

$$
\frac{i}{2} \not \partial W_{+}=(m-\not k) W_{+},
$$

which proves that (4.8) solves the (3.4). The form in the eq. (4.8) can be further simplified. Defining the tilde transform of $\beta$ from the eq. (1.2) as in (4.2)

$$
\widetilde{\beta}(\varpi)=\sum_{k=0}^{\infty} \frac{i^{k}}{(k+1) !}(\underbrace{\varpi_{\alpha_{1}}^{\mu} \varpi_{\alpha_{2}}^{\alpha_{1}} \ldots \varpi^{\alpha_{k-1}} \alpha_{k}}_{k \text { times }}) \beta^{\alpha_{k}},
$$

and taking advantage of the two identities proved in ref. [1], namely:

$$
\sum_{k=1}^{n} \Lambda^{-k} \widetilde{b}(\varpi)=n \widetilde{b}(-n \varpi)
$$

and

$$
-i x \cdot\left(\Lambda^{n} p-p\right)-n \widetilde{b}(-n \varpi) \cdot p=-n \widetilde{\beta}(-n \varpi) \cdot p,
$$

we can rewrite the (4.8) as:

$$
\begin{aligned}
W(x, k) & =\frac{1}{(2 \pi)^{3}} \int \frac{\mathrm{d}^{3} \mathrm{p}}{2 \varepsilon} \sum_{n=1}^{\infty}(-1)^{n+1} \mathrm{e}^{-n \widetilde{\beta}_{n} \cdot p} \times \\
& {\left[S(\Lambda)^{n}(m+\not p) \delta^{4}\left(k-\frac{\Lambda^{n} p+p}{2}\right)+(m-\not p) S(\Lambda)^{-n} \delta^{4}\left(k+\frac{\Lambda^{n} p+p}{2}\right)\right], }
\end{aligned}
$$

where, henceforth:

$$
\widetilde{\beta}_{n} \equiv \widetilde{\beta}(-n \varpi) .
$$

This is our final expression for the covariant Wigner function for imaginary thermal vorticity; for the massless case, the expression is obtained by just taking the $m=0$ limit of the (4.10). Like the expectation values (4.5), it cannot straightforwardly be continued to real thermal vorticity because of the singular delta functions. Nevertheless, the (4.10) can be integrated in either $\mathrm{d}^{4} k$ or $\mathrm{d}^{3} \mathrm{p}$ and the resulting expressions are fit to be continued analytically.

\section{Currents at global thermodynamic equilibrium}

The mean currents (3.5) are integrals of the Wigner function (4.10) and, as such, we will see that they give rise to non-singular expressions. Let us start by studying the vector current $j^{\mu}(x)$. Of course, in case of vanishing chemical potential, as implied by $\zeta=0$, it ought to be zero and yet it is instructive too see how this comes about. Plugging the (4.10) into the (3.5a) we obtain:

$$
j^{\mu}(x)=\frac{1}{(2 \pi)^{3}} \int \frac{\mathrm{d}^{3} \mathrm{p}}{2 \varepsilon} \sum_{n=1}^{\infty}(-1)^{n+1} \mathrm{e}^{-n \widetilde{\beta}_{n} \cdot p}\left[\operatorname{tr}\left(\gamma^{\mu} S(\Lambda)^{n} \not p\right)-\operatorname{tr}\left(\gamma^{\mu} \not p S(\Lambda)^{-n}\right)\right] .
$$


The first term is the particle contribution, whereas the second term is the antiparticle one. Now, by using known properties of the trace and of the $\gamma^{0} \mathcal{C}$ matrix:

$$
\begin{aligned}
\operatorname{tr}\left(\gamma^{\mu} \not p S(\Lambda)^{-n}\right) & =\operatorname{tr}\left(\left(\gamma^{\mu} \not p S(\Lambda)^{-n}\right)^{T}\right) \\
& =\operatorname{tr}\left(S(\Lambda)^{\left.n T^{-1} \not p^{T} \gamma^{\mu T}\right)}\right. \\
& =\operatorname{tr}\left(\gamma^{0} \mathcal{C} S(\Lambda)^{n} \mathcal{C} \gamma^{0} \gamma^{0} \mathcal{C} \not p \mathcal{C} \gamma^{0} \gamma^{0} \mathcal{C} \gamma^{\mu} \mathcal{C} \gamma^{0}\right) \\
& =\operatorname{tr}\left(\gamma^{\mu} S(\Lambda)^{n} \not p\right)
\end{aligned}
$$

which proves that the integrand in (5.1) vanishes.

Using the commutation rules of the gamma matrices, the particle term of the trace in (5.1) can be written as:

$$
\operatorname{tr}\left(\not p \gamma^{\mu} S(\Lambda)^{n}\right)=p^{\mu} \operatorname{tr}\left(S(\Lambda)^{n}\right)+2 i p_{\nu} \operatorname{tr}\left(\Sigma^{\mu \nu} S(\Lambda)^{n}\right),
$$

therefore, the particle term of the vector current is:

$$
j_{+}^{\mu}(x)=\frac{1}{(2 \pi)^{3}} \int \frac{\mathrm{d}^{3} \mathrm{p}}{2 \varepsilon} \sum_{n=1}^{\infty}(-1)^{n+1} \mathrm{e}^{-n \widetilde{\beta}_{n} \cdot p}\left[p^{\mu} \operatorname{tr}\left(S(\Lambda)^{n}\right)+2 i p_{\nu} \operatorname{tr}\left(\Sigma^{\mu \nu} S(\Lambda)^{n}\right)\right],
$$

where we can identify a term proportional to $p^{\mu}$ and one perpendicular to it (see the discussion at the end of section 3 and in section 8).

The series for the axial current (3.5b) can be derived likewise. In this case, being the axial current even under charge conjugation, it may be non vanishing with $\zeta=0$. Indeed, it can be seen that the particle and antiparticle contributions sum up; from the eq. (4.10):

$$
j_{A}^{\mu}(x)=\frac{2}{(2 \pi)^{3}} \int \frac{\mathrm{d}^{3} \mathrm{p}}{2 \varepsilon} \sum_{n=1}^{\infty}(-1)^{n+1} \mathrm{e}^{-n \widetilde{\beta}_{n} \cdot p_{\operatorname{tr}}}\left(\gamma^{\mu} \gamma_{5} S(\Lambda)^{n} \not p\right) .
$$

Like in (5.3) the trace can be split:

$$
\operatorname{tr}\left(\not p \gamma^{\mu} \gamma_{5} S(\Lambda)^{n}\right)=p^{\mu} \operatorname{tr}\left(\gamma_{5} S(\Lambda)^{n}\right)+2 i p_{\nu} \operatorname{tr}\left(\Sigma^{\mu \nu} \gamma_{5} S(\Lambda)^{n}\right) .
$$

It is convenient, for later use, to introduce a compact notation and define:

$$
\begin{aligned}
& A^{\mu \nu}(n)=\operatorname{tr}\left(\gamma^{\nu} \gamma^{\mu} S(\Lambda)^{n}\right)=g^{\mu \nu} \operatorname{tr}\left(S(\Lambda)^{n}\right)+2 i \operatorname{tr}\left(\Sigma^{\mu \nu} S(\Lambda)^{n}\right), \\
& A_{5}^{\mu \nu}(n)=\operatorname{tr}\left(\gamma^{\nu} \gamma^{\mu} \gamma_{5} S(\Lambda)^{n}\right)=g^{\mu \nu} \operatorname{tr}\left(\gamma_{5} S(\Lambda)^{n}\right)+2 i \operatorname{tr}\left(\Sigma^{\mu \nu} \gamma_{5} S(\Lambda)^{n}\right) .
\end{aligned}
$$

and rewrite the axial current accordingly:

$$
\begin{aligned}
j_{A}^{\mu}(x) & =\frac{2}{(2 \pi)^{3}} \int \frac{\mathrm{d}^{3} \mathrm{p}}{2 \varepsilon} \sum_{n=1}^{\infty}(-1)^{n+1} \mathrm{e}^{-n \widetilde{\beta}_{n} \cdot p} \operatorname{tr}\left(\gamma^{\mu} \gamma_{5} S(\Lambda)^{n} \not p\right) \\
& =\frac{2}{(2 \pi)^{3}} \int \frac{\mathrm{d}^{3} \mathrm{p}}{2 \varepsilon} \sum_{n=1}^{\infty}(-1)^{n+1} \mathrm{e}^{-n \widetilde{\beta}_{n} \cdot p} p_{\nu} A_{5}^{\mu \nu}(n) \\
& =\frac{2}{(2 \pi)^{3}} \sum_{n=1}^{\infty} \frac{(-1)^{n+1}}{n} A_{5}^{\mu \nu}(n)\left(-\frac{\partial}{\partial \widetilde{\beta}_{n}^{\nu}}\right) \int \frac{\mathrm{d}^{3} \mathrm{p}}{2 \varepsilon} \mathrm{e}^{-n \widetilde{\beta}_{n} \cdot p} .
\end{aligned}
$$


The uniform convergence of the series of the integrands [1] for imaginary thermal vorticity makes it possible to exchange the series with the integration. In the massless case, the momentum integral is more easily carried out and one gets:

$$
\int \frac{\mathrm{d}^{3} \mathrm{p}}{2 \varepsilon} \mathrm{e}^{-n \widetilde{\beta}_{n} \cdot p}=\frac{2 \pi}{n^{2} \widetilde{\beta}_{n} \cdot \widetilde{\beta}_{n}},
$$

so that the (5.7) becomes:

$$
j_{A}^{\mu}(x)=\frac{1}{\pi^{2}} \sum_{n=1}^{\infty} \frac{(-1)^{n+1}}{n^{3}} \frac{\widetilde{\beta}_{n \nu}}{\left(\widetilde{\beta}_{n}\right)^{4}} A_{5}^{\mu \nu}(n) .
$$

The quantum statistics series can be obtained for the stress-energy tensor as well. By using (4.10) in the definition (3.5c), one has:

$$
\begin{aligned}
T_{C}^{\mu \nu} & =\int \mathrm{d}^{4} k k^{\nu} \operatorname{tr}\left(\gamma^{\mu} W(x, k)\right) \\
& =\sum_{n=1}^{\infty} \frac{(-1)^{n+1}}{(2 \pi)^{3}} \int \frac{\mathrm{d}^{3} \mathrm{p}}{2 \varepsilon} \mathrm{e}^{-n \tilde{\beta}_{n} \cdot p}\left[\left(\frac{\Lambda^{n} p+p}{2}\right)^{\nu} \operatorname{tr}\left(\gamma^{\mu} S(\Lambda)^{n} \not p\right)+\left(\frac{\Lambda^{n} p+p}{2}\right)^{\nu} \operatorname{tr}\left(\gamma^{\mu} \not p S(\Lambda)^{-n}\right)\right] \\
& =\sum_{n=1}^{\infty} \frac{2(-1)^{n+1}}{(2 \pi)^{3}} \int \frac{\mathrm{d}^{3} \mathrm{p}}{2 \varepsilon} \mathrm{e}^{-n \tilde{\beta}_{n} \cdot p}\left(\frac{\Lambda^{n} p+p}{2}\right)^{\nu} \operatorname{tr}\left(\gamma^{\mu} S(\Lambda)^{n} \not p\right),
\end{aligned}
$$

where the eq. (5.2) has been used. Now the momenta can be expressed as derivatives with respect to $\widetilde{\beta}_{n}$ and, by using the shorthand (5.5):

$$
T_{C}^{\mu \nu}=\sum_{n=1}^{\infty} \frac{(-1)^{n+1}}{(2 \pi)^{3} n^{2}}\left(A^{\mu \alpha}(n) \frac{\partial}{\partial \widetilde{\beta}_{n \nu}} \frac{\partial}{\partial \widetilde{\beta}_{n}^{\alpha}}+\left(\Lambda^{n}\right)_{\rho}^{\nu} A^{\mu \alpha}(n) \frac{\partial}{\partial \widetilde{\beta}_{n \rho}} \frac{\partial}{\partial \widetilde{\beta}_{n}^{\alpha}}\right) \int \frac{\mathrm{d}^{3} \mathrm{p}}{2 \varepsilon} \mathrm{e}^{-n \widetilde{\beta}_{n} \cdot p} .
$$

In the massless case, the momentum integral (5.8) is simple and the above series can be written as:

$$
T_{C}^{\mu \nu}=\sum_{n=1}^{\infty} \frac{(-1)^{n+1}}{2 \pi^{2}} \frac{1}{n^{4} \widetilde{\beta}_{n}^{4}}\left[A_{\gamma}^{\mu}(n)\left(4 \frac{\widetilde{\beta}_{n}^{\nu} \widetilde{\beta}_{n}^{\gamma}}{\widetilde{\beta}_{n}^{2}}-g^{\nu \gamma}\right)+\left(\Lambda^{n}\right)_{\rho}^{\nu} A_{\gamma}^{\mu}(n)\left(4 \frac{\widetilde{\beta}_{n}^{\rho} \widetilde{\beta}_{n}^{\gamma}}{\widetilde{\beta}_{n}^{2}}-g^{\rho \gamma}\right)\right] .
$$

All of the above quantum statistics series have been derived for imaginary $\varpi$, but they no longer feature, unlike the Wigner function (4.10), singular terms and they can, in principle, be continued analytically to the physical real $\varpi$. It is indeed possible to obtain finite expressions by Taylor expanding each term of the series in $\varpi$ at some fixed order, continue to real $\varpi$ and sum the series in $n$ at each order in $\varpi$. However, just as for the scalar field [1], it can be realized that this method does not eventually lead to a finite result. Indeed, the full series in $n$ are actually divergent, so the above method of expanding in $\varpi$ and resumming the partial series in $n$ term by term generates an asymptotic expansion in $\varpi$. The ultimate reason for the lack of convergence of the full, quantum statistic series has been deeply investigated in [1] and it is twofold: on the one hand, for pure rotation, the exponential of the density operator $\widehat{H}-\omega / T_{0} \widehat{J}_{z}$ is not bounded from below unless a boundary condition on the field is set at some finite radius $r<1 / \omega$; on the other hand, 
for pure acceleration, the iterative method which led to the (4.5) and ensuing expressions introduces spurious, non-analytic contributions which must be subtracted. In order to obtain finite exact results, before analytically continuing to real $\varpi$, it is necessary to extract the analytic part of the functions of $\varpi$ around $\varpi=0$ by means of the procedure of analytic distillation introduced in ref. [1].

\section{Fermionic analytic distillation}

The building block of all the expressions found so far is the solution (4.5) of the equation (4.4), which was obtained by iteration. However, as it was discussed in ref. [1], this solution may not be unique. In general, a solution of the (4.4) is the sum of the general solution of the corresponding homogeneous equation

$$
\left\langle\widehat{a}_{s}^{\dagger}(p) \widehat{a}_{t}\left(p^{\prime}\right)\right\rangle=(-1)^{2 S} \sum_{r} D^{S}(W(\Lambda, p))_{r s} \mathrm{e}^{-\widetilde{b} \cdot \Lambda p}\left\langle\widehat{a}_{r}^{\dagger}(\Lambda p) \widehat{a}_{t}(p)\right\rangle
$$

and a special solution of the (4.4). It can be shown that the homogeneous equation cannot have analytic solutions in $\phi=i \varpi=0$ [1]. Nevertheless, it may have non-analytic solutions and, specifically, they do occur in the pure acceleration case, while in the pure rotation case there is no non-trivial solution of the homogeneous equation. Hence, unless we deal with the pure rotation case, the one analytic solution of the (4.4) can be determined by subtracting the non-analytic contributions in $\phi=i \varpi=0$.

The mathematical method to make such a subtraction was introduced in ref. [1] and was named analytic distillation. The definition reads:

Definition. Let $f(z)$ be a function on a domain $D$ of the complex plane and $z_{0} \in \bar{D}$ a point where the function may not be analytic. Suppose that asymptotic ${ }^{2}$ power series of $f(z)$ in $z-z_{0}$ exist in subsets $D_{i} \subset D$ such that $\cup_{i} D_{i}=D$ :

$$
f(z) \sim \sum_{n} a_{n}^{(i)}\left(z-z_{0}\right)^{n}
$$

where $n$ can take integer negative values. If the series formed with the common coefficients in the various subsets restricted to $n \geq 0$ has a positive radius of convergence, the analytic function defined by this power series is called analytic distillate of $f(z)$ in $z_{0}$ and it is denoted by $\operatorname{dist}_{z_{0}} f(z)$.

As it is apparent from the above definition, analytic distillation requires the existence of an asymptotic power series of the function at the point of interest. However, all series in section 5 are neither convergent power series nor asymptotic power series, rather formal series of trigonometric and hyperbolic functions of $n$-dependent argument (see section 7). In the scalar field case [1], where one deals with series of the form:

$$
g(x)=\sum_{n} f(n x)
$$

\footnotetext{
${ }^{2}$ We denote asymptotic equality with the symbol $\sim$.
} 
a theorem due to Zagier [31, 32] allows to obtain an asymptotic power series of the function $g(x)$ :

$$
g(x) \sim \frac{I_{f}}{x}+\sum_{n=0}^{\infty} a_{n} \zeta(-n) x^{n}, \quad I_{f}=\int_{0}^{\infty} \mathrm{d} x f(x),
$$

once the asymptotic power series of $f$ is known:

$$
f(x) \sim \sum_{n=0}^{\infty} a_{n} x^{n} .
$$

However, as it is apparent in section 5, the fermionic series are of the form:

$$
g(x)=\sum_{n}(-1)^{n+1} f(n x),
$$

and Zagier's theorem does not apply. To handle asymptotic expansions of such alternating series, it is thus necessary to extend Zagier's theorem and the asymptotic formula. For this purpose, the appropriate tool is the generalized Mellin transform, as envisaged in ref. [31].

Given a function $\varphi(x)$, its Mellin transform is defined as:

$$
\{\mathcal{M} \varphi\}(s)=\int_{0}^{\infty} \mathrm{d} t \varphi(t) t^{s-1}
$$

whence:

$$
\{\mathcal{M} \varphi(\lambda x)\}(s)=\lambda^{-s}\{\mathcal{M} \varphi\}(s) \quad \lambda \in \mathbb{R}_{>0} .
$$

The Mellin transform defines an holomorphic function of $s$ if $\varphi(x)$ decays rapidly both at zero and infinity. If these requirements are not fulfilled, the Mellin transform defines an holomorphic function in a smaller region of the complex plane. For instance, if $\varphi(t) \rightarrow t^{-A}$ as $t \rightarrow 0$ and $\varphi(t) \rightarrow t^{-B}$ as $t \rightarrow \infty$, the Mellin transform is holomorphic in the strip $A<s<B$. In such cases, we can make a meromorphic continuation of the Mellin transform over a larger domain. For instance, assume $\varphi$ to be a function of rapid decay at infinity but to have the following asymptotic expansion in 0 :

$$
\varphi(x) \sim \sum_{n=0} a_{n} x^{n}
$$

In this case the Mellin transform defined in (6.2) exists only in the half-plane Re $s>0$. However we can analytically continue the transformed function by defining for some $N>0$ :

$$
\{\mathcal{M} \varphi\}(s)=\int_{0}^{1} \mathrm{~d} t\left(\varphi(t)-\sum_{n=0}^{N-1} a_{n} t^{n}\right) t^{s-1}+\sum_{n=0}^{N-1} \frac{a_{n}}{n+s}+\int_{1}^{\infty} \mathrm{d} t \varphi(t) t^{s-1} .
$$

This new definition extends Mellin transforms over a larger region of the complex plane Re $s>-N$, and shows that such a continuation is a meromorphic function with simple poles in $s=-n$ with residue $a_{n}$. Conversely, if the generalized Mellin transformation $\{\mathcal{M} \varphi\}$ is a meromorphic function with simple poles in $s=-n$, then the coefficients of the asymptotic power series of $\varphi$ are the residues of $\{\mathcal{M} \varphi\}$ [32]. These are the basic features we need for the purpose of this work, for further information on the generalized Mellin transformation we refer the reader to the ref. [31] and [32]. 
We are now in the position to prove the theorem:

Theorem 1. Let $F(z)$ be a $C^{\infty}$ complex valued function in a domain of the complex plane and suppose that $F$ has the following asymptotic power series in $z=0$

$$
F(z) \sim \sum_{k=-M}^{\infty} A_{k} z^{k}
$$

with $M$ a positive integer and let $F$ be $o(1 /|z|)$ when $|z| \rightarrow+\infty$ in the real axis. Let $G(z)$ be the function defined by the series:

$$
G(z)=\sum_{n=1}^{\infty}(-1)^{n+1} F(n z) .
$$

The asymptotic power series of $G(z)$ for $|z| \rightarrow 0^{+}$, is given by:

$$
G(z) \sim \sum_{n=-M}^{\infty} A_{n} \eta(-n) z^{n}
$$

where $\eta$ is the Dirichlet function:

$$
\eta(s)=\sum_{k=1}^{\infty} \frac{(-1)^{k+1}}{k^{s}}=\left(1-2^{1-s}\right) \zeta(s) .
$$

To prove the theorem, we first consider a complex valued function with real argument. Consider then $f$ on the positive real axis with the following asymptotic power series in $x=0$ :

$$
f(x) \sim \sum_{k=-M}^{\infty} a_{k} x^{k}
$$

and let us define the function $g$ as

$$
g(x)=\sum_{n=1}^{\infty}(-1)^{n+1} f(n x) .
$$

It can be shown that $g$ has the following asymptotic power series about $0^{+}$:

$$
g(x) \sim \sum_{n=-M}^{\infty} a_{n} \eta(-n) x^{n} .
$$

To prove it, let us first remove the negative-powers terms of the asymptotic series of $f$ by defining two new functions $\tilde{f}$ and $\tilde{g}$ :

$$
\tilde{f}(x)=f(x)-\sum_{k=-M}^{-1} a_{k} x^{k}, \quad \tilde{g}(x)=\sum_{n=1}^{\infty}(-1)^{n+1} \tilde{f}(n x) .
$$

It readily follows that

$$
g(x)=\tilde{g}(x)+\sum_{n=1}^{\infty} \sum_{k=-M}^{-1}(-1)^{n+1} a_{k}(n x)^{k} .
$$


The finite and infinite sum can be exchanged, yielding:

$$
g(x)=\tilde{g}(x)+\sum_{k=-M}^{-1} a_{k} \eta(-k) x^{k} .
$$

We now move to the asymptotic expansion of $\tilde{g}$. From the properties of the generalized Mellin transform, $\{\mathcal{M} \tilde{f}\}$ is a meromorphic function having poles in 0 and in all the negative integers, with residues $a_{n}$. Let us now consider the Mellin transform of $\tilde{g}$ :

$$
\{\mathcal{M} \tilde{g}\}(s)=\sum_{n=1}^{\infty}(-1)^{n+1}\{\mathcal{M} \tilde{f}(n x)\}=\sum_{n=1}^{\infty}(-1)^{n+1} n^{-s}\{\mathcal{M} \tilde{f}\}(s)=\eta(s)\{\mathcal{M} \tilde{f}\}(s) .
$$

Since the $\eta$ function is holomorphic in the complex plane, then $\{\mathcal{M} \tilde{g}\}(s)$ is a meromorphic function having simple poles in zero and in all negative integers, just as $\{\mathcal{M} \tilde{f}\}$, with residues $\eta(-n) a_{n}$. Therefore, according to the theorem proved in ref. [32], $\tilde{g}(t)$ must have the following power asymptotic expansion about zero:

$$
\tilde{g}(x) \sim \sum_{n=0}^{\infty} a_{n} \eta(-n) x^{n} .
$$

Plugging the asymptotic expansion of $\tilde{g}$ in (6.5) we finally obtain the asymptotic expansion of $g$ :

$$
g(x) \sim \sum_{n=-M}^{\infty} a_{n} \eta(-n) x^{n},
$$

which proves the theorem for a function of real argument. This result can be extended to a function of complex variable $F(z)$ with an asymptotic power series about $z=0$ :

$$
F(z) \sim \sum_{n=-M}^{\infty} A_{n} z^{n}
$$

Defining:

$$
x=|z| \Longrightarrow z=x \mathrm{e}^{i \varphi},
$$

and a new complex-valued function $f$ of the real variable $x$ with fixed $\varphi$ :

$$
f(x) \equiv F\left(x \mathrm{e}^{i \varphi}\right) .
$$

Now, $f$ has an asymptotic expansion about $x=0$ given by:

$$
f(x) \sim \sum_{n=-M}^{\infty} A_{n} \mathrm{e}^{i n \varphi} x^{n} \equiv \sum_{n=-M}^{\infty} a_{n} x^{n} .
$$

From the previous proof, it follows:

$$
\begin{aligned}
G(z) & \equiv \sum_{n=1}(-1)^{n+1} F(n z)=\sum_{n=1}(-1)^{n+1} F\left(n x \mathrm{e}^{i \varphi}\right)=\sum_{n=1}(-1)^{n+1} f(n x) \\
& \sim \sum_{n=-M}^{\infty} a_{n} \eta(-n) x^{n}=\sum_{n=-M}^{\infty} A_{n} \eta(-n) z^{n}
\end{aligned}
$$

which proves the theorem in its complete form. 
We can conclude that, if the asymptotic expansion (6.4) is convergent, the analytic distillate of $G$ is:

$$
\operatorname{dist}_{0} G(z)=\operatorname{dist}_{0} \sum_{n=1}^{\infty}(-1)^{n+1} F(n z)=\sum_{n=0}^{\infty} A_{n} \eta(-n) z^{n} .
$$

The basic idea of the method established by this theorem is to expand in a power series of $z$ the function $F$ in the series (6.3), to then force the exchange of the two series, and to finally replace the divergent expressions with the analytic continuations of the $\eta$ Dirichlet function. A remarkable difference with the non-alternating case (6.1), is the absence of the term proportional to $x^{-1}$; this is owing to the non-singular behaviour of the $\eta$ function in $s=1$, at variance with the $\zeta$. This has an interesting consequence, namely that the full asymptotic series of the function $G(z)$ in (6.3) can be obtained in practice by expanding $f(z)$ in power series (including negative powers) around $z=0$, exchange the series and inserting the $\eta$ function; in fact, this procedure misses the integral term in the scalar case [32]. It is worth mentioning that the $\eta$ function vanishes for all negative even numbers, so one can often end up with a finite sum in the distillate.

\section{Exact mean values of currents at global thermodynamic equilibrium}

We are now in a position to analytically continue the series obtained in section 5 to real thermal vorticity and to obtain finite results by applying the analytic distillation. We begin with the pure acceleration case and continue with pure rotation and, finally, with a more general case.

\subsection{Acceleration}

As extensively studied elsewhere [24, 25, 33-37], thermoydnamic equilibrium with constant proper acceleration (along the $z$-axis) is characterized by the following thermal vorticity and four-temperature field:

$$
\varpi_{\mu \nu}=\frac{a}{T_{0}}\left(g_{3 \mu} g_{0 \nu}-g_{0 \mu} g_{3 \nu}\right), \quad \beta^{\mu}=\frac{1}{T_{0}}(1+a z, 0,0, a t) .
$$

In this case one can calculate the mean values of scalar operators at $t=z=0$ and obtain them in a general space-time point by means of the simple substitution $\beta(0) \rightarrow \beta(x)[1]$. For imaginary thermal vorticity, setting $\phi=i a / T_{0}$ we have:

$$
n \widetilde{\beta}_{n}^{\mu}(t=0, z=0)=\left(\frac{\sinh (n \phi)}{T_{0} \phi}, 0,0, \frac{1-\cosh (n \phi)}{T_{0} \phi}\right),
$$

hence:

$$
\beta(0)^{2}=\frac{1}{T_{0}^{2}}, \quad n^{2} \widetilde{\beta}_{n}^{2}=\beta(0)^{2} \frac{4 \sinh ^{2}\left(\frac{n \phi}{2}\right)}{\phi^{2}} .
$$


Using the above expressions, the form of a Lorentz transformation, $S(\Lambda)$ from (2.6) and the eq. (5.5) (5.6), we obtain the following matrices:

$$
\begin{aligned}
& \left(\Lambda^{n}\right)^{\mu}{ }_{\nu}=\left(\begin{array}{cccc}
\cosh n \phi & 0 & 0 & \sinh n \phi \\
0 & 1 & 0 & 0 \\
0 & 0 & 1 & 0 \\
\sinh n \phi & 0 & 0 & \cosh n \phi
\end{array}\right), \quad S(\Lambda)^{n}=\left(\begin{array}{cccc}
\mathrm{e}^{n \phi / 2} & 0 & 0 & 0 \\
0 & \mathrm{e}^{-n \phi / 2} & 0 & 0 \\
0 & 0 & \mathrm{e}^{-n \phi / 2} & 0 \\
0 & 0 & 0 & \mathrm{e}^{n \phi / 2}
\end{array}\right), \\
& A^{\mu \nu}=\left(\begin{array}{cccc}
4 \cosh \frac{n \phi}{2} & 0 & 0 & -4 \sinh \frac{n \phi}{2} \\
0 & -4 \cosh \frac{n \phi}{2} & 0 & 0 \\
0 & 0 & -4 \cosh \frac{n \phi}{2} & 0 \\
4 \sinh \frac{n \phi}{2} & 0 & 0 & -4 \cosh \frac{n \phi}{2}
\end{array}\right), \quad A_{5}^{\mu \nu}=\left(\begin{array}{cccc}
0 & 0 & 0 & 0 \\
0 & 0 & 4 i \sinh \frac{n \phi}{2} & 0 \\
0 & -4 i \sinh \frac{n \phi}{2} & 0 & 0 \\
0 & 0 & 0 & 0
\end{array}\right) \text {. }
\end{aligned}
$$

which, once entered into the eq. (5.10), provide us with the stress-energy tensor of a free massless Dirac field in $x=0$ with imaginary acceleration $a=-i \phi T_{0}$. Henceforth, the series in $\phi$ for mean values will be denoted with an additional subscript $I$, for instance:

$$
T_{C}^{00}(0)_{I}=\frac{3}{8 \pi^{2}} \frac{1}{\beta^{4}} \sum_{n=1}^{\infty}(-1)^{n+1} \phi^{4} \frac{\sinh n \phi}{\sinh ^{5}\left(\frac{n \phi}{2}\right)} \equiv \frac{3}{8 \pi^{2}} \frac{1}{\beta^{4}} S_{F, 4}(\phi) .
$$

The series $S_{F, 4}(\phi)$ uniformly converges when $\phi$ has a non-vanishing real part, whereas in the physical case of imaginary $\phi$ the series is badly divergent because of the sine function in the denominator, thus analytic distillation is required. The analytic distillation of a general class of series of the type in eq. (7.4) are accurately studied in the appendix B; here we sketch the specific procedure for the series in eq. (7.4). The asymptotic power series of the function $T_{C}^{00}(0)_{I}$ can be obtained by using theorem 1 . The first step is to derive an asymptotic power series expansion about zero of the function to be summed, what can be obtained by writing the Laurent series of the ratio

$$
\frac{\sinh \phi}{\sinh ^{5} \frac{\phi}{2}}=\frac{32}{\phi^{4}}-\frac{4}{3 \phi^{2}}-\frac{17}{180}+\sum_{n=1}^{\infty} a_{n} \phi^{2 n}
$$

which holds true for any complex $\phi$. Since all the positive powers are even, the vanishing of the $\eta$-function in the asymptotic formula of theorem 1 yields a polynomial:

$$
S_{F, 4}(\phi)=\phi^{4} \sum_{n=1}^{\infty}(-1)^{n+1} \frac{\sinh n \phi}{\sinh ^{5}\left(\frac{n \phi}{2}\right)} \sim \phi^{4}\left(\frac{32 \eta(4)}{\phi^{4}}-\frac{4 \eta(2)}{3 \phi^{2}}-\frac{17 \eta(0)}{180}\right) .
$$

Now that we have a converging asymptotic expansion, the analytic distillate of the series is

$$
\operatorname{dist}_{\phi=0} T_{C}^{00}(0)_{I}=\operatorname{dist}_{\phi=0}\left(\frac{3}{8 \pi^{2}} \frac{1}{\beta^{4}} S_{F, 4}(\phi)\right)=\frac{7 \pi^{2}}{60 \beta^{4}}-\frac{\phi^{2}}{24 \beta^{4}}-\frac{17 \phi^{4}}{960 \pi^{2} \beta^{4}} .
$$

The same method can be used for the other components and one eventually obtains:

$$
\begin{aligned}
\operatorname{dist}_{\phi=0} T_{C}^{\text {off diag }} & =0, \\
\operatorname{dist}_{\phi=0} T_{C}^{11 ; 22 ; 33} & =\operatorname{dist}_{\phi=0}\left(\frac{1}{8 \pi^{2}} \frac{1}{\beta^{4}} S_{F, 4}(\phi)\right)=\frac{7 \pi^{2}}{180 \beta^{4}}-\frac{\phi^{2}}{72 \beta^{4}}-\frac{17 \phi^{4}}{2880 \pi^{2} \beta^{4}}
\end{aligned}
$$


The analytic distillation yields simple polynomials and the analytic continuation to real acceleration is readily done by replacing $\phi \rightarrow i a / T_{0}$.

From these components, we can find the thermodynamic coefficients appearing in the general decomposition of the energy-momentum tensor at equilibrium with acceleration $[1,35]$ :

$$
T_{C}^{\mu \nu}=\rho u^{\mu} u^{\nu}-p\left(g^{\mu \nu}-u^{\mu} u^{\nu}\right)+\mathcal{A} \alpha^{\mu} \alpha^{\nu},
$$

where $u^{\mu}=\beta^{\mu} / \sqrt{\beta^{2}}$ and $\alpha^{\mu}=\varpi^{\mu \nu} u_{\nu}$ like in eq. (1.3). After the continuation to the physical case, we find:

$$
\begin{aligned}
& \rho=\frac{7 \pi^{2}}{60 \beta^{4}}-\frac{\alpha^{2}}{24 \beta^{4}}-\frac{17 \alpha^{4}}{960 \pi^{2} \beta^{4}}, \\
& p=\frac{7 \pi^{2}}{180 \beta^{4}}-\frac{\alpha^{2}}{72 \beta^{4}}-\frac{17 \alpha^{4}}{2880 \pi^{2} \beta^{4}}, \\
& \mathcal{A}=0
\end{aligned}
$$

where $\alpha^{2}=\alpha^{\mu} \alpha_{\mu}=-a^{2} / T_{0}^{2}$. According to the remark below the equation (7.1), making $\beta^{2}$ coordinate-dependent extend the validity of the above expressions to all space-time.

The results (7.5) are in agreement with the perturbative expansion to second order in acceleration of refs. [24, 34] and to fourth order of refs. [36-38]. A further confirmation that these are the actual exact result is their vanishing at the Unruh temperature $T_{U}=$ $a / 2 \pi[33,36]$. We note that these formulae upgrade those found in ref. [39] which were based on an approximated form of the Wigner function at equilibrium. Therein, a puzzling result was found, that is the vanishing of the energy density at $T_{0}=a / \pi$ instead of the Unruh temperature $a / 2 \pi$. With the exact form of the Wigner function found, the agreement with Unruh temperature is fully restored. This feature extends to a large class of local operators, see appendix B. Since their mean values are obtained through integration of the Wigner function, we argue that the vanishing at the Unruh temperature must extend to any integral of the Wigner function (4.10), after analytic distillation and continuation.

\subsection{Rotation}

We now turn to the global thermodynamic equilibrium with pure rotation, characterized by:

$$
\varpi_{\mu \nu}=\frac{\omega}{T_{0}}\left(g_{\mu 1} g_{\nu 2}-g_{\mu 2} g_{\nu 1}\right), \quad \beta^{\mu}=\frac{1}{T_{0}}(1,-\omega y, \omega x, 0),
$$

where $\omega$ is the constant angular velocity. Thus, the density operator of rotational thermodynamic equilibrium reads:

$$
\widehat{\rho}=\frac{1}{Z} \exp \left[-\frac{\widehat{H}}{T_{0}}+\frac{1}{T_{0}} \boldsymbol{\omega} \cdot \widehat{\mathbf{J}}\right] .
$$

The tilde-transformed four-temperature vector for imaginary angular velocity (i.e. $\left.\omega / T_{0}=-i \phi\right)$ is:

$$
n \widetilde{\beta}(i n \phi)=\left(\frac{n}{T_{0}}, i x(\cos (n \phi)-1)+i y \sin (n \phi), i y(\cos (n \phi)-1)-i x \sin (n \phi), 0\right),
$$


and its squared magnitude is:

$$
n^{2} \widetilde{\beta}_{n}^{2}=\frac{n^{2}}{T_{0}^{2}}+4 r^{2} \sin ^{2}\left(\frac{n \phi}{2}\right),
$$

where $r^{2}=x^{2}+y^{2}$. From (7.6), (2.6) and (5.5), (5.6) we obtain:

$$
\begin{aligned}
\left(\Lambda^{n}\right)^{\mu}{ }_{\nu} & =\left(\begin{array}{cccc}
1 & 0 & 0 & 0 \\
0 & \cos n \phi & -\sin n \phi & 0 \\
0 & \sin n \phi & \cos n \phi & 0 \\
0 & 0 & 0 & 1
\end{array}\right), \quad S(\Lambda)^{n}=\left(\begin{array}{ccccc}
\mathrm{e}^{-i n \phi / 2} & 0 & 0 & 0 \\
0 & \mathrm{e}^{i n \phi / 2} & 0 & 0 \\
0 & 0 & \mathrm{e}^{-i n \phi / 2} & 0 \\
0 & 0 & 0 & \mathrm{e}^{i n \phi / 2}
\end{array}\right), \\
A^{\mu \nu} & =\left(\begin{array}{ccccccc}
4 \cos \left(\frac{n \phi}{2}\right) & 0 & 0 & 0 \\
0 & -4 \cos \left(\frac{n \phi}{2}\right) & 4 \sin \left(\frac{n \phi}{2}\right) & 0 \\
0 & -4 \sin \left(\frac{n \phi}{2}\right) & -4 \cos \left(\frac{n \phi}{2}\right) & 0 \\
0 & 0 & 0 & -4 \cos \left(\frac{n \phi}{2}\right)
\end{array}\right), \quad A_{5}^{\mu \nu}=\left(\begin{array}{ccccc}
0 & 0 & 4 i \sin \frac{n \phi}{2} \\
0 & 0 & 0 \\
0 & 0 & 0 & 0 \\
-4 i \sin \frac{n \phi}{2} & 0 & 0 & 0
\end{array}\right) .
\end{aligned}
$$

As an example, we calculate the stress-energy tensor. For comparison with known results in the literature, we calculate the symmetrized Belinfante stress-energy tensor, which, for the Dirac field, has a simple relation with the previously quoted canonical one:

$$
T_{B}^{\mu \nu}(x)=\frac{1}{2}\left(T_{C}^{\mu \nu}(x)+T_{C}^{\nu \mu}(x)\right),
$$

and it is thus easy to derive it from (5.10). For instance, plugging the (7.8) and (7.9) in the 00-component of (5.10) we obtain:

$$
T_{B}^{00}(x)_{I}=\frac{1}{2 \pi^{2}} \sum_{n=1}^{\infty} \frac{(-1)^{n+1} 8 T_{0}^{4} \cos \left(\frac{n \phi}{2}\right)\left[3 n^{2}+2 r^{2} T_{0}^{2}(\cos n \phi-1)\right]}{\left(n^{2}+4 r^{2} T_{0}^{2} \sin ^{2}\left(\frac{n \phi}{2}\right)\right)^{3}} .
$$

The series is convergent for real $\phi$, but it becomes divergent as soon as $\phi$ gets a small imaginary part, just like in the scalar field case [1] (the denominator features infinitely many zeroes, and the series becomes densely divergent in the complex plane). Indeed, without boundary condition of the field at a finite radius $r$ such that $\omega r<1[1]$, the operator $\hat{H}-\omega \widehat{J}_{z}$ in the eq. (7.7) is not bounded from below and gives rise to unphysical divergences. Thus, while the analytic distillation procedure leads to finite results, the physical meaning of rotation without boundary conditions remains limited [40].

Moreover, in the distillation of the function in eq. (7.11), and for all the series likewise obtained from the Wigner function (4.10) at thermodynamic equilibrium with rotation, there is an additional difficulty. Indeed, as it appears from (7.11), the series does not depend on the product $n \phi$ alone and thus we cannot straightforwardly apply the theorem 1 . To overcome this problem, we use a method that we proposed in ref. [1]. The first step is to introduce an auxiliary real parameter $B$, and replacing $\phi$ with $B$ any time the former is not accompanied by a factor $n$. For the (7.11):

$$
T_{B}^{00}(x)_{I}=\frac{1}{2 \pi^{2}} \sum_{n=1}^{\infty} \lim _{B \rightarrow \phi} \frac{(-1)^{n+1} 8 B^{4} T_{0}^{4} \cos \left(\frac{n \phi}{2}\right)\left[3 n^{2} \phi^{2}+2 r^{2} B^{2} T_{0}^{2}(\cos n \phi-1)\right]}{\left(n^{2} \phi^{2}+4 r^{2} B^{2} T_{0}^{2} \sin ^{2}\left(\frac{n \phi}{2}\right)\right)^{3}} .
$$


Since the series is a uniformly convergent series of continuous functions of $\phi$ and $B$, for real $B$ and $\phi$, one can exchange the limit with the sum and obtain a series which is now suitable for the application of the theorem 1 :

$$
T_{B}^{00}(x)_{I}=\lim _{B \rightarrow \phi} \frac{1}{2 \pi^{2}} \sum_{n=1}^{\infty} \frac{(-1)^{n+1} 8 B^{4} T_{0}^{4} \cos \left(\frac{n \phi}{2}\right)\left[3 n^{2} \phi^{2}+2 r^{2} B^{2} T_{0}^{2}(\cos n \phi-1)\right]}{\left(n^{2} \phi^{2}+4 r^{2} B^{2} T_{0}^{2} \sin ^{2}\left(\frac{n \phi}{2}\right)\right)^{3}} .
$$

The asymptotic power series obtained with theorem 1 is indeed a finite polynomial in $\phi$ :

$$
\begin{aligned}
T_{B}^{00}(x)_{I} \sim & \lim _{B \rightarrow \phi} \frac{7 \pi^{2} B^{4} T_{0}^{4}}{60 \phi^{4}\left(1+B^{2} r^{2} T_{0}^{2}\right)^{3}}-\frac{7 \pi^{2} B^{6} r^{2} T_{0}^{6}}{180 \phi^{4}\left(1+B^{2} r^{2} T_{0}^{2}\right)^{3}}+\frac{7 B^{6} r^{2} T_{0}^{6}}{36 \phi^{2}\left(1+B^{2} r^{2} T_{0}^{2}\right)^{4}} \\
& -\frac{B^{8} r^{4} T_{0}^{8}}{72 \phi^{2}\left(1+B^{2} r^{2} T_{0}^{2}\right)^{4}}-\frac{B^{4} T_{0}^{4}}{8 \phi^{2}\left(1+B^{2} r^{2} T_{0}^{2}\right)^{4}}+\frac{17 B^{10} r^{6} T_{0}^{10}}{2880 \pi^{2}\left(1+B^{2} r^{2} T_{0}^{2}\right)^{5}} \\
& +\frac{247 B^{8} r^{4} T_{0}^{8}}{2880 \pi^{2}\left(1+B^{2} r^{2} T_{0}^{2}\right)^{5}}-\frac{137 B^{6} r^{2} T_{0}^{6}}{576 \pi^{2}\left(1+B^{2} r^{2} T_{0}^{2}\right)^{5}}+\frac{B^{4} T_{0}^{4}}{64 \pi^{2}\left(1+B^{2} r^{2} T_{0}^{2}\right)^{5}}
\end{aligned}
$$

Now, one can take the limit $B \rightarrow \phi$, thereby obtaining a series which implicitly provides the asymptotic power series of the original function (7.11) about $\phi=0$, taking into account the analyticity of the various expressions in $B$ in (7.12) [1]. We can then apply analytic distillation and continue the result to the physical angular velocity $\phi \rightarrow i \omega / T_{0}$. The final expression is:

$$
\begin{aligned}
T_{B}^{00}(x)= & \frac{7}{180} \pi^{2}\left(4 \gamma^{2}-1\right) \gamma^{4} T_{0}^{4}+\frac{1}{72}\left(24 \gamma^{4}-16 \gamma^{2}+1\right) \gamma^{4} T_{0}^{2} \omega^{2}+ \\
& +\frac{\left(960 \gamma^{6}-1128 \gamma^{4}+196 \gamma^{2}+17\right) \gamma^{4} \omega^{4}}{2880 \pi^{2}}
\end{aligned}
$$

where we defined:

$$
\gamma=\frac{1}{\sqrt{1-r^{2} \omega^{2}}}
$$

This procedure can be carried out for all the components of the Belinfante stress-energy tensor (see appendix C); here we just quote the final results.

For this purpose, it is convenient to introduce a tetrad of orthogonal, non-normalized vectors:

$$
u^{\mu}=\frac{\beta^{\mu}}{\sqrt{\beta^{2}}}, \quad \alpha^{\mu}=\varpi^{\mu \nu} u_{\nu}, \quad w^{\mu}=-\frac{1}{2} \epsilon^{\mu \nu \rho \sigma} \varpi_{\nu \rho} u_{\sigma}, \quad l^{\mu}=\epsilon^{\mu \nu \rho \sigma} w_{\nu} \alpha_{\rho} u_{\sigma},
$$

and to express the variables $T_{0}, \omega$ and $R^{2}$ in terms of the Lorentz invariants:

$$
\beta^{2}=\frac{1}{\gamma^{2} T_{0}^{2}}, \quad \alpha^{2}=-\left(\gamma^{2}-1\right) \frac{\omega^{2}}{T_{0}^{2}} w^{2}=-\gamma^{2} \frac{\omega^{2}}{T_{0}^{2}}, \quad l^{2}=-\alpha^{2} w^{2} .
$$


Also, we can decompose the Belinfante stress-energy tensor along the tetrad (7.13), with eleven scalar thermodynamic coefficients ${ }^{3}$ as follows:

$$
\begin{aligned}
T_{B}^{\mu \nu}(x)= & \rho u^{\mu} u^{\nu}-p \Delta^{\mu \nu}+W w^{\mu} w^{\nu}+A \alpha^{\mu} \alpha^{\nu}+G^{l} l^{\mu} l^{\nu}+G\left(l^{\mu} u^{\nu}+l^{\nu} u^{\mu}\right)+\mathbb{A}\left(\alpha^{\mu} u^{\nu}+\alpha^{\nu} u^{\mu}\right) \\
& +G^{\alpha}\left(l^{\mu} \alpha^{\nu}+l^{\nu} \alpha^{\mu}\right)+\mathbb{W}\left(w^{\mu} u^{\nu}+w^{\nu} u^{\mu}\right)+A^{w}\left(\alpha^{\mu} w^{\nu}+\alpha^{\nu} w^{\mu}\right)+G^{w}\left(l^{\mu} w^{\nu}+l^{\nu} w^{\mu}\right) .
\end{aligned}
$$

To unambiguously identify all terms, we first calculate:

$$
T_{B}^{\mu \nu} \frac{l_{\nu}}{l^{2}} \frac{l_{\mu}}{l^{2}}=G^{l} l^{2}-p=\frac{17 \alpha^{4}}{2880 \pi^{2} \beta^{4}}+\frac{\alpha^{2}}{72 \beta^{4}}-\frac{7 \pi^{2}}{180 \beta^{4}}-\frac{w^{4}}{192 \pi^{2} \beta^{4}}+\frac{11 w^{2} \alpha^{2}}{160 \pi^{2} \beta^{4}}+\frac{w^{2}}{24 \beta^{4}},
$$

and, taking into account that of $l^{2}=-\alpha^{2} w^{2}$, we can single out $G^{l}$ and $p$. The remaining terms are easily identified:

$$
\begin{aligned}
\rho & =\frac{7 \pi^{2}}{60 \beta^{4}}-\frac{\alpha^{2}}{24 \beta^{4}}-\frac{w^{2}}{8 \beta^{4}}-\frac{17 \alpha^{4}}{960 \pi^{2} \beta^{4}}+\frac{w^{4}}{64 \pi^{2} \beta^{4}}+\frac{23 \alpha^{2} w^{2}}{1440 \pi^{2} \beta^{4}}, \\
p & =\frac{7 \pi^{2}}{180 \beta^{4}}-\frac{\alpha^{2}}{72 \beta^{4}}-\frac{w^{2}}{24 \beta^{4}}-\frac{17 \alpha^{4}}{2880 \pi^{2} \beta^{4}}+\frac{w^{4}}{192 \pi^{2} \beta^{4}}, \\
G^{l} & =-\frac{11}{160 \pi^{2} \beta^{4}}, \\
G & =\frac{1}{18 \beta^{4}}-\frac{31 \alpha^{2}}{360 \pi^{2} \beta^{4}}-\frac{13 w^{2}}{120 \pi^{2} \beta^{4}}, \\
W & =-\frac{61 \alpha^{2}}{1440 \pi^{2} \beta^{4}}, \\
A & =-\frac{61 w^{2}}{1440 \pi^{2} \beta^{4}}, \\
\mathbb{A} & =\mathbb{W}=G^{\alpha}=G^{w}=A^{w}=0 .
\end{aligned}
$$

The above coefficients coincide with those calculated in [41-43] by solving the Dirac equation in cylindrical coordinates. In ref. [43], series similar to those in eq. (7.11) and in the appendix $\mathrm{C}$ have been studied, but a different regularization scheme has been used. We stress that, like for the scalar field [1], after setting $w^{\mu}=0$, the results (7.15) reduce to the (7.5) of equilibrium with pure acceleration.

We conclude this section by reporting the exact mean value of the axial current. For a massless field the axial current is given by the imaginary thermal vorticity series (5.9). The analytic distillation and the subsequent analytic continuation can be carried out the same way as for the series (7.11) (see appendix C for details), and one obtains:

$$
j_{\mathrm{A}}^{\mu}=\frac{1}{\beta^{2}}\left(\frac{1}{6}-\frac{w^{2}}{24 \pi^{2}}-\frac{\alpha^{2}}{8 \pi^{2}}\right) \frac{w^{\mu}}{\sqrt{\beta^{2}}} .
$$

Again, this result coincides with previous results evaluated with both perturbative [24, $34,44]$ and exact $[41,42,45]$ methods. Note that the time-reversal symmetry prevents

\footnotetext{
${ }^{3}$ There is a redundancy due to the fact that on the rotation axis the defined tetrad is no longer a basis, see the discussion in [1].
} 
the axial current to be directed along the acceleration vector. Instead, the induced axial current along the vorticity of the system is an effect which became known as Axial Vortical Effect [46]. This effect is allowed by the symmetry of the density operator and is expected for both massive [34] and massless Dirac fields at equilibrium. Unlike the electric current induced by the rotation, the axial vortical effect can contain terms (see eq. (7.16)) independent of the axial and chemical potentials and that are not constrained by the chiral anomaly [47].

\subsection{Thermodynamic equilibrium with rotation and acceleration}

Having successfully tested the fermionic analytic distillation method by comparing its output with the results known in literature and otherwise determined, we can apply it to calculate yet unknown expressions. Particularly, the global thermodynamic equilibrium with both rotation and acceleration along the $z$-axis has never been explored, as it is difficult to define the appropriate curvilinear coordinates and solve the relevant field equations. In fact, the analytic distillation method, as we are going to show, is much more convenient.

We thus proceed to study the thermodynamic equilibrium with both rotation and acceleration along $z$-axis for the free Dirac field, extending the results obtained for the scalar field in ref. [1]. This is described by the density operator (1.1) with a thermal vorticity and temperature given by:

$$
\varpi_{\mu \nu}=\frac{\omega}{T_{0}}\left(g_{\mu 1} g_{\nu 2}-g_{\nu 1} g_{\mu 2}\right)+\frac{a}{T_{0}}\left(g_{\mu 3} g_{\nu 0}-g_{\nu 3} g_{\mu 0}\right) \quad \beta^{\mu}=\frac{1}{T_{0}}(1+a z,-\omega y, \omega x, a t) .
$$

Defining once again the tetrad $\left\{u^{\mu}, \alpha^{\mu}, w^{\mu}, l^{\mu}\right\}$ as in eq. (7.13), we first observe that, unlike the previous cases, the scalar $\alpha \cdot w=-a \omega / T_{0}^{2}$ is non-vanishing and can thus appear in the mean values.

As a case study, we determine the full Belinfante stress-energy tensor for massless fermions. The tilde-transformed four-temperature vector reads:

$$
\begin{aligned}
n \widetilde{\beta}_{n}= & \left(i t\left(\cos \left(\frac{a n}{T_{0}}\right)-1\right)+\frac{(1+a z) \sin \left(\frac{a n}{T_{0}}\right)}{a},-y \sinh \left(\frac{n \omega}{T_{0}}\right)+i x\left(\cosh \left(\frac{n \omega}{T_{0}}\right)-1\right),\right. \\
& \left.x \sinh \left(\frac{n \omega}{T_{0}}\right)+i y\left(\cosh \left(\frac{n \omega}{T_{0}}\right)-1\right), t \sin \left(\frac{a n}{T_{0}}\right)+\frac{i(1+a z)\left(\cos \left(\frac{a n}{T_{0}}\right)-1\right)}{a}\right),
\end{aligned}
$$

and, after replacing $a / T_{0} \rightarrow-i \Phi, \omega / T_{0} \rightarrow-i \phi$ the additional matrices appearing in the 
series (5.10) are:

$$
\begin{aligned}
\Lambda_{\nu}^{\mu} & =\left(\begin{array}{cccc}
\cosh n \Phi & 0 & 0 & \sinh n \Phi \\
0 & \cos n \phi & -\sin n \phi & 0 \\
0 & \sin n \phi & \cos n \phi & 0 \\
\sinh n \Phi & 0 & 0 & \cosh n \Phi
\end{array}\right), \\
S(\Lambda)^{n} & =\left(\begin{array}{cccc}
\mathrm{e}^{n(\Phi-i \phi) / 2} & 0 & 0 & 0 \\
0 & \mathrm{e}^{-n(\Phi-i \phi) / 2} & 0 & 0 \\
0 & 0 & \mathrm{e}^{-n(\Phi+i \phi) / 2} & 0 \\
0 & 0 & 0 & \mathrm{e}^{n(\Phi+i \phi) / 2}
\end{array}\right), \\
A^{\mu \nu} & =\left(\begin{array}{cccc}
4 \cos \left(\frac{n \phi}{2}\right) \cosh \left(\frac{n \Phi}{2}\right) & 0 & 0 & -4 \cos \left(\frac{n \phi}{2}\right) \sinh \left(\frac{n \Phi}{2}\right) \\
0 & -4 \cos \left(\frac{n \phi}{2}\right) \cosh \left(\frac{n \Phi}{2}\right) & 4 \sin \left(\frac{n \phi}{2}\right) \cosh \left(\frac{n \Phi}{2}\right) & 0 \\
0 & -4 \sin \left(\frac{n \phi}{2}\right) \cosh \left(\frac{n \Phi}{2}\right) & -4 \cos \left(\frac{n \phi}{2}\right) \cosh \left(\frac{n \Phi}{2}\right) & 0 \\
4 \cos \left(\frac{n \phi}{2}\right) \sinh \left(\frac{n \Phi}{2}\right) & 0 & 0 & -4 \cos \left(\frac{n \phi}{2}\right) \cosh \left(\frac{n \Phi}{2}\right)
\end{array}\right), \\
A_{5}^{\mu \nu} & =\left(\begin{array}{cccc}
-4 i \sin \frac{n \phi}{2} \sinh \frac{n \Phi}{2} & 0 & 0 & 4 i \sin \frac{n \phi}{2} \cosh \frac{n \Phi}{2} \\
0 & 4 i \sin \frac{n \phi}{2} \sinh \frac{n \Phi}{2} & 4 i \cos \frac{n \phi}{2} \sinh \frac{n \Phi}{2} & 0 \\
0 & -4 i \cos \frac{n \phi}{2} \sinh \frac{n \Phi}{2} & 4 i \sin \frac{n \phi}{2} \sinh \frac{n \Phi}{2} & 0 \\
4 i \sin \frac{n \phi}{2} \cosh \frac{n \Phi}{2} & 0 & 0 & 4 i \sin \frac{n \phi}{2} \sinh \frac{n \Phi}{2}
\end{array}\right) .
\end{aligned}
$$

The analytic distillation of the series is described in appendix D. The coefficients of the general decomposition (7.14) turn out to be:

$$
\begin{aligned}
\rho & =\frac{7 \pi^{2}}{60 \beta^{4}}-\frac{\alpha^{2}}{24 \beta^{4}}-\frac{w^{2}}{8 \beta^{4}}-\frac{17 \alpha^{4}}{960 \pi^{2} \beta^{4}}+\frac{w^{4}}{64 \pi^{2} \beta^{4}}+\frac{23 \alpha^{2} w^{2}}{1440 \pi^{2} \beta^{4}}+\frac{11(\alpha \cdot w)^{2}}{720 \pi^{2} \beta^{4}}, \\
p & =\frac{7 \pi^{2}}{180 \beta^{4}}-\frac{\alpha^{2}}{72 \beta^{4}}-\frac{w^{2}}{24 \beta^{4}}-\frac{17 \alpha^{4}}{2880 \pi^{2} \beta^{4}}+\frac{w^{4}}{192 \pi^{2} \beta^{4}}+\frac{(\alpha \cdot w)^{2}}{96 \pi^{2} \beta^{4}}, \\
G & =-\frac{11}{160 \pi^{2} \beta^{4}}, \\
G & =\frac{1}{18 \beta^{4}}-\frac{31 \alpha^{2}}{360 \pi^{2} \beta^{4}}-\frac{13 w^{2}}{120 \pi^{2} \beta^{4}}, \\
W & =-\frac{61 \alpha^{2}}{1440 \pi^{2} \beta^{4}}, \\
A & =-\frac{61 w^{2}}{1440 \pi^{2} \beta^{4}}, \\
A^{w} & =\frac{61 \alpha \cdot w}{1440 \pi^{2} \beta^{4}}, \\
\mathbb{A} & =\mathbb{W}=G^{\alpha}=G^{w}=0 .
\end{aligned}
$$

These coefficients are consistent with those in eq. (7.15), with regard to their dependence on the scalars $\alpha^{2}$ and $w^{2}$. The actual payoff of this case is to have found out the dependence of the coefficients on the scalar $\alpha \cdot w$, which was vanishing in both the pure rotation and the pure acceleration cases. We have also calculated the mean axial current, whose expression coincides with (7.16) with no extra dependence on $\alpha \cdot w$ (see appendix D). 


\section{Massless particles and the chiral kinetic theory}

Over the last decade, there has been considerable interest in the relativistic kinetic theory of massless fermions, known as chiral kinetic theory [10-12]. The common approach to this problem is based on a semi-classical expansion in $\hbar$ of the Wigner equation and an educated ansatz of the equilibrium distribution function. In this section, after reviewing the definition of the distribution function, we obtain exact expressions at global thermodynamic equilibrium.

Because of the decoupling between left and right currents, we can rewrite the particle term of the mean current (3.6) for massless particles as:

$$
j_{+}^{\mu}(x)=\frac{1}{(2 \pi)^{3}} \sum_{\lambda} \int \frac{\mathrm{d}^{3} \mathrm{p}}{2 \varepsilon} \frac{\mathrm{d}^{3} \mathrm{p}^{\prime}}{2 \varepsilon^{\prime}} \mathrm{e}^{i\left(p^{\prime}-p\right) \cdot x}\left\langle\widehat{a}_{\lambda}^{\dagger}\left(p^{\prime}\right) \widehat{a}_{\lambda}(p)\right\rangle \bar{u}_{\lambda}\left(p^{\prime}\right) \gamma^{\mu} u_{\lambda}(p)
$$

where $\lambda$ is the helicity, taking values $-1 / 2$ and $1 / 2$. The spinorial product can be calculated (see appendix A), yielding:

$$
\bar{u}_{\lambda}\left(p^{\prime}\right) \gamma^{\mu} u_{\lambda}(p)=\frac{1}{\sqrt{p \cdot \overline{\mathfrak{p}} p^{\prime} \cdot \overline{\bar{p}}}}\left(p^{\mu} p^{\prime} \cdot \overline{\mathfrak{p}}+p^{\prime \mu} p \cdot \overline{\mathfrak{p}}-p \cdot p^{\prime} \overline{\mathfrak{p}}^{\mu}+2 i \lambda \epsilon^{\mu \rho \sigma \tau} p_{\rho}^{\prime} p_{\sigma} \overline{\mathfrak{p}}_{\tau}\right) .
$$

Instead of replacing the above expression in the (8.1), we try to identify a projection of the right hand side of the equation (8.2) onto the four-vector $p$, in accordance to the methods of chiral kinetic theory. We first observe that $L^{\mu}=\bar{u}_{\lambda}\left(p^{\prime}\right) \gamma^{\mu} u_{\lambda}(p)$ is a complex light-like vector, just by squaring the right hand side of (8.2). Therefore, it can be decomposed onto two light-like vectors $p$ and $q$ such that $p \cdot q \neq 0$, and a third vector $N$ which is orthogonal to both $p$ and $q$ :

$$
L^{\mu}=\frac{L \cdot q}{q \cdot p} p^{\mu}+\frac{L \cdot p}{q \cdot p} q^{\mu}+N^{\mu}(p, q, L)
$$

(this can be taken as a definition of $N$ ). Now, because of the Dirac equation, $L \cdot p=0$, and we are left with:

$$
\bar{u}_{\lambda}\left(p^{\prime}\right) \gamma^{\mu} u_{\lambda}(p)=\frac{\bar{u}_{\lambda}\left(p^{\prime}\right) q u_{\lambda}(p)}{q \cdot p} p^{\mu}+N^{\mu} .
$$

Using the above decomposition into the current (8.1) we obtain:

$$
\begin{aligned}
j_{+}^{\mu}(x)= & \frac{1}{(2 \pi)^{3}} \sum_{\lambda} \int \frac{\mathrm{d}^{3} \mathrm{p}}{\varepsilon} p^{\mu} \int \frac{\mathrm{d}^{3} \mathrm{p}^{\prime}}{2 \varepsilon^{\prime}} \frac{\bar{u}_{\lambda}\left(p^{\prime}\right) \phi u_{\lambda}(p)}{2 q \cdot p} \mathrm{e}^{i\left(p^{\prime}-p\right) \cdot x}\left\langle\widehat{a}_{\lambda}^{\dagger}\left(p^{\prime}\right) \widehat{a}_{\lambda}(p)\right\rangle \\
& +\frac{1}{(2 \pi)^{3}} \sum_{\lambda} \int \frac{\mathrm{d}^{3} \mathrm{p}}{\varepsilon} N^{\mu} \int \frac{\mathrm{d}^{3} \mathrm{p}^{\prime}}{4 \varepsilon^{\prime}} \mathrm{e}^{i\left(p^{\prime}-p\right) \cdot x}\left\langle\widehat{a}_{\lambda}^{\dagger}\left(p^{\prime}\right) \widehat{a}_{\lambda}(p)\right\rangle .
\end{aligned}
$$

The above equation defines a decomposition of the current in phase space $\mathcal{J}^{\mu}(p, x)$ (see equation (3.7)) onto the on-shell light-like vector $p$ and a vector $N$ which is perpendicular to it. To achieve it, we had to introduce an arbitrary light-like vector $q$; while the decomposition is inevitably dependent on it, the current is altogether independent thereof once all terms are included. Following chiral kinetic theory, the decomposition (8.1) allows to 
define an on-shell $q$-dependent distribution function $f_{\lambda}(x, p)_{(q)}$ as the coefficient of $p^{\mu}$ in the decomposition of $\mathcal{J}^{\mu}(p, x)$ :

$$
f_{\lambda}(x, p)_{(q)} \equiv \frac{1}{(2 \pi)^{3}} \int \frac{\mathrm{d}^{3} \mathrm{p}^{\prime}}{2 \varepsilon^{\prime}} \mathrm{e}^{i\left(p^{\prime}-p\right) \cdot x}\left\langle\widehat{a}_{\lambda}^{\dagger}\left(p^{\prime}\right) \widehat{a}_{\lambda}(p)\right\rangle \frac{\bar{u}_{\lambda}\left(p^{\prime}\right) q u_{\lambda}(p)}{2 q \cdot p} .
$$

A suitable choice of $q$ is just the standard vector $\mathfrak{p}$; in this case, the (8.3) becomes, by using the (8.2):

$$
f_{\lambda}(x, p)_{(\mathfrak{p})}=\frac{1}{(2 \pi)^{3}} \int \frac{\mathrm{d}^{3} \mathrm{p}^{\prime}}{2 \varepsilon^{\prime}} \sqrt{\frac{p^{\prime} \cdot \overline{\mathfrak{p}}}{p \cdot \overline{\mathfrak{p}}}} \mathrm{e}^{i\left(p^{\prime}-p\right) \cdot x}\left\langle\widehat{a}_{\lambda}^{\dagger}\left(p^{\prime}\right) \widehat{a}_{\lambda}(p)\right\rangle .
$$

The form (8.3) is especially suitable to obtain an exact expression. Using trace cyclicity we can write $\bar{u}_{\lambda}\left(p^{\prime}\right) q u_{\lambda}(p)=\operatorname{tr}\left(q u_{\lambda}(p) \bar{u}_{\lambda}\left(p^{\prime}\right)\right)$. Dealing with massless spinors, we can isolate the helicity lambda using the chiral projectors:

$$
\begin{aligned}
f_{\lambda}(x, p)_{(q)} & =\frac{1}{(2 \pi)^{3}} \int \frac{\mathrm{d}^{3} \mathrm{p}^{\prime}}{2 \varepsilon^{\prime}} \mathrm{e}^{i\left(p^{\prime}-p\right) \cdot x}\left\langle\widehat{a}_{\lambda}^{\dagger}\left(p^{\prime}\right) \widehat{a}_{\lambda}(p)\right\rangle \frac{\operatorname{tr}\left(q u_{\lambda}(p) \bar{u}_{\lambda}\left(p^{\prime}\right)\right)}{2 q \cdot p} \\
& =\frac{1}{(2 \pi)^{3}} \int \frac{\mathrm{d}^{3} \mathrm{p}^{\prime}}{2 \varepsilon^{\prime}} \frac{\mathrm{e}^{i\left(p^{\prime}-p\right) \cdot x}}{2 q \cdot p} \sum_{s, t} \operatorname{tr}\left(q \frac{I+2 \lambda \gamma_{5}}{2}\left\langle\widehat{a}_{t}^{\dagger}\left(p^{\prime}\right) \widehat{a}_{s}(p)\right\rangle u_{s}(p) \bar{u}_{t}\left(p^{\prime}\right) \frac{I-2 \lambda \gamma_{5}}{2}\right) \\
& =\frac{1}{(2 \pi)^{3}} \frac{1}{2 q \cdot p} \operatorname{tr}\left(q \frac{I+2 \lambda \gamma_{5}}{2} \int \frac{\mathrm{d}^{3} \mathrm{p}^{\prime}}{2 \varepsilon^{\prime}} \mathrm{e}^{i\left(p^{\prime}-p\right) \cdot x} \sum_{s, t}\left\langle\widehat{a}_{t}^{\dagger}\left(p^{\prime}\right) \widehat{a}_{s}(p)\right\rangle u_{s}(p) \bar{u}_{t}\left(p^{\prime}\right)\right),
\end{aligned}
$$

where we used the anticommutation rules of $\gamma_{5}$ and $\gamma_{5} u_{\lambda}(p)=2 \lambda u_{\lambda}(p)$, where $\lambda$ is the helicity and $2 \lambda$ the chirality. Also note that $(2 \lambda)^{2}=1$.

Except for $q$ and the chiral projector, the expression in the trace, and in particular the product $\left\langle\widehat{a}_{t}^{\dagger}\left(p^{\prime}\right) \widehat{a}_{s}(p)\right\rangle u_{s}(p) \bar{u}_{t}\left(p^{\prime}\right)$, is very similar to the particle part of the Wigner function (3.2). The substitution of the exact result (4.5) and the subsequent calculations can be done as in section 4.2 , and one gets:

$$
\int \frac{\mathrm{d}^{3} \mathrm{p}^{\prime}}{2 \varepsilon^{\prime}} \mathrm{e}^{i\left(p^{\prime}-p\right) \cdot x} \sum_{s, t}\left\langle\widehat{a}_{t}^{\dagger}\left(p^{\prime}\right) \widehat{a}_{s}(p)\right\rangle u_{s}(p) \bar{u}_{t}\left(p^{\prime}\right)=\sum_{n=1}^{\infty}(-1)^{n+1} \mathrm{e}^{-n \widetilde{\beta}_{n} \cdot p} S(\Lambda)^{n}(\not p+m) .
$$

Plugging this result in the trace above, we have:

$$
f_{\lambda}(x, p)_{(q)}=\frac{1}{(2 \pi)^{3}} \frac{1}{2 p \cdot q} \sum_{n=1}(-1)^{n+1} \mathrm{e}^{-n \widetilde{\beta}_{n} \cdot p} \operatorname{tr}\left(\frac{I+2 \lambda \gamma_{5}}{2} S(\Lambda)^{n} \not p q q\right) .
$$

Expanding the $\not p q$ product, we obtain the final expression of the exact equilibrium distribution function:

$$
\begin{aligned}
f_{\lambda}(x, p)_{(q)}= & \operatorname{dist}_{\varpi=0} \sum_{n=1}^{\infty} \frac{(-1)^{n+1}}{2(2 \pi)^{3}} \mathrm{e}^{-n \widetilde{\beta}(-n \varpi) \cdot p}\left\{\operatorname{tr}\left(\frac{I+2 \lambda \gamma_{5}}{2} \exp \left[n \frac{\varpi_{\rho \sigma}}{2} \Sigma^{\rho \sigma}\right]\right)\right. \\
& \left.+\frac{2 i q_{\mu} p_{\nu}}{q \cdot p} \operatorname{tr}\left(\frac{I+2 \lambda \gamma_{5}}{2} \Sigma^{\mu \nu} \exp \left[n \frac{\varpi_{\rho \sigma}}{2} \Sigma^{\rho \sigma}\right]\right)\right\},
\end{aligned}
$$

where the real thermal vorticity has been introduced in the series, provided that it is made convergent by the analytic distillation in $\varpi=0$. This expression gives rise to a current term along $p^{\mu}$ which is in agreement with the general expression (5.4). 
Applying analytic distillation to the (8.4) goes beyond the scope of this work. Indeed, the theorem 1 cannot be applied straightforwardly as the series is not of the required functional form, so a new method to obtain the full asymptotic power series in $\varpi$ is needed. The (8.4) differs from the usual ansatz of the equilibrium distribution [12, 15, 16, 21] mostly for the exponential factor and for the absence of the frame vector.

\section{Spin density matrix and spin polarization vector}

As has been mentioned in the introduction, there is a phenomenological considerable interest on spin and polarization in relativistic fluids [9]. In the context of heavy ion collisions, the vorticity of the quark gluon plasma is transferred to the quasi free fermions (e.g. the $\Lambda$ particles) during hadronization, resulting in a non-vanishing polarization of hadrons. Our method provides the opportunity to calculate an exact expression of the spin polarization vector and the spin density matrix at global equilibrium with acceleration and rotation. Such expressions are still unknown and the formulae used in literature are just the leading order term in the thermal vorticity. Even though thermal vorticity is a small quantity in all phenomenological applications, it is important to know the exact expression to estimate the quantitative impact of higher order terms.

The spin density matrix — for a free field — is defined in a quantum field theoretical framework as:

$$
\Theta_{r s}(p)=\frac{\operatorname{Tr}\left(\widehat{\rho} \widehat{a}_{s}^{\dagger}(p) \widehat{a}_{r}(p)\right)}{\sum_{t} \operatorname{Tr}\left(\widehat{\rho} \widehat{a}_{t}^{\dagger}(p) \widehat{a}_{t}(p)\right)},
$$

where $r, s, t$ are the spin states labels; this matrix is the full description of the spin state of a particle. For massive particles, the spin density matrix and the spin polarization vector can be expressed by means of the Wigner function as [48]:

$$
\begin{aligned}
\Theta(p) & =\frac{\int \mathrm{d} \Sigma_{\mu} p^{\mu} \bar{U}(p) W_{+}(x, p) \mathrm{U}(p)}{\operatorname{tr}_{2}\left(\int \mathrm{d} \Sigma_{\mu} p^{\mu} \bar{U}(p) W_{+}(x, p) \mathrm{U}(p)\right)}, \\
S^{\mu}(p) & =\frac{1}{2} \frac{\int \mathrm{d} \Sigma \cdot p \operatorname{tr}\left(\gamma^{\mu} \gamma_{5} W_{+}(x, p)\right)}{\int \mathrm{d} \Sigma \cdot p \operatorname{tr}\left(W_{+}(x, p)\right)}
\end{aligned}
$$

where the integration over the (arbitrary) space-like hypersurface $\Sigma$ with the measure $\mathrm{d} \Sigma \cdot p$ makes the momentum $p$ on-shell. This is a general feature for free fields [48] and we are going to explicitly prove it for the Wigner function $W(x, k)$ found in (4.10). First of all, one has:

$$
k^{\mu} \partial_{\mu} W_{ \pm}(x, k)=0 .
$$

To show it, one should keep in mind that from (4.9) one has

$$
-n \widetilde{\beta}(-n \varpi) \cdot p=-n \widetilde{b}(-n \varpi) \cdot p-i x \cdot\left(\Lambda^{n} p-p\right),
$$

so that, taking the derivatives of the exponent in (4.10) the following factors are obtained:

$$
k \cdot\left(\left(\Lambda^{n} p-p\right)\right) \delta^{4}\left(k-\frac{\Lambda^{n} p+p}{2}\right), \quad k \cdot\left(\left(\Lambda^{n} p-p\right)\right) \delta^{4}\left(k+\frac{\Lambda^{n} p+p}{2}\right),
$$


which both vanish, proving the (9.3). Now, provided that suitable boundary conditions apply, we can then calculate the integral:

$$
\int_{\Sigma} \mathrm{d} \Sigma_{\mu} k^{\mu} W_{+}(x, k)
$$

over any hypersurface, and particularly the hypersurface $t=0$. We thus obtain, by integrating the (4.10) in $\mathrm{d}^{3} x$, a factor $\delta^{3}(\mathbf{k}-\mathbf{p})$ in the particle term of the Wigner function, hence:

$$
\int_{\Sigma} \mathrm{d} \Sigma_{\mu} k^{\mu} W_{+}(x, k)=\delta\left(k^{0}-\varepsilon_{k}\right) \frac{1}{2} \sum_{n=1}^{\infty}(-1)^{n+1} \mathrm{e}^{-n \widetilde{b}(-n \varpi) \cdot k} S(\Lambda)^{n}(m+\not k)
$$

which makes $k$ manifestly on shell.

Therefore, by using the (9.4) with $k=p$, and continuing the Wigner function to real thermal vorticity (with associated distillation) the (9.1) gives rise to:

$$
\Theta(p)=\operatorname{dist}_{\varpi=0} \frac{\sum_{n=1}^{\infty}(-1)^{n+1} \mathrm{e}^{-n \widetilde{b}(-n \varpi) \cdot p} \overline{\mathrm{U}}(p) \exp [n \varpi: \Sigma / 2] \mathrm{U}(p)}{\operatorname{tr}_{2} \sum_{n=1}^{\infty}(-1)^{n+1} \mathrm{e}^{-n \widetilde{b}(-n \varpi) \cdot p} \overline{\mathrm{U}}(p) \exp [n \varpi: \Sigma / 2] \mathrm{U}(p)},
$$

where the Dirac equation for the spinors $U$ has been used. Similarly, the spin polarization vector (9.2) at global thermodynamic equilibrium becomes:

$$
\begin{aligned}
S^{\mu}(p) & =\operatorname{dist}_{\varpi=0} \frac{1}{2 m} \frac{\sum_{n=1}^{\infty}(-1)^{n+1} \mathrm{e}^{-n \widetilde{b}(-n \varpi) \cdot p} \operatorname{tr}\left(\gamma^{\mu} \gamma_{5} \exp [n \varpi: \Sigma / 2] \not p\right)}{\sum_{n=1}^{\infty}(-1)^{n+1} \mathrm{e}^{-n \widetilde{b}(-n \varpi) \cdot p} \operatorname{tr}(\exp [n \varpi: \Sigma / 2])} \\
& =\operatorname{dist}_{\varpi=0} \frac{1}{2 m} \frac{\sum_{n=1}^{\infty}(-1)^{n+1} \mathrm{e}^{-n \widetilde{b}(-n \varpi) \cdot p} p_{\nu} A_{5}^{\mu \nu}(n, \varpi)}{\sum_{n=1}^{\infty}(-1)^{n+1} \mathrm{e}^{-n \widetilde{b}(-n \varpi) \cdot p} \operatorname{tr}(\exp [n \varpi: \Sigma / 2])},
\end{aligned}
$$

where we used (5.6) for real thermal vorticity. For the global equilibrium with pure acceleration, described in the subsection 7.1, we have:

$$
\mathrm{e}^{n \varpi: \Sigma / 2}=\left(\begin{array}{cccc}
\mathrm{e}^{i n a / 2 T_{0}} & 0 & 0 & 0 \\
0 & \mathrm{e}^{-i n a / 2 T_{0}} & 0 & 0 \\
0 & 0 & \mathrm{e}^{-i n a / 2 T_{0}} & 0 \\
0 & 0 & 0 & \mathrm{e}^{i n a / 2 T_{0}}
\end{array}\right) \quad A_{5}^{\mu \nu}=\left(\begin{array}{cccc}
0 & 0 & 0 & 0 \\
0 & 0 & -4 \sin \frac{n a}{2 T_{0}} & 0 \\
0 & 4 \sin \frac{n a}{2 T_{0}} & 0 & 0 \\
0 & 0 & 0 & 0
\end{array}\right) \text {. }
$$

Hence, at the Unruh temperature $T_{0}=a / 2 \pi$, the first matrix becomes the identity and the tensor $A_{5}$ vanishes, making the spin polarization vector in eq. (9.6) zero. This is in excellent agreement with the expectations from the physics of the Unruh effect, which implies the equivalence of the Minkowski vacuum (with, of course, no polarization) at the finite temperature $a / 2 \pi$ for an accelerated observer.

As discussed at the end of section 5 , it is possible to obtain a finite full expression at linear order in real thermal vorticity by expanding in $\varpi$ :

$$
\operatorname{tr}\left(\gamma^{\mu} \gamma_{5} S(\Lambda)^{n} \not p\right) \sim-n \varpi_{\alpha \beta} p_{\nu} \epsilon^{\mu \alpha \beta \nu}+\mathcal{O}\left(\varpi^{2}\right), \quad \mathrm{e}^{-n \widetilde{b}(-n \varpi) \cdot p} \sim e^{-b \cdot p}+\mathcal{O}(\varpi),
$$


and summing the series in $n$. The result is:

$$
S^{\mu}(p) \sim-\frac{1}{8 m} \epsilon^{\mu \alpha \beta \nu} \varpi_{\alpha \beta} p_{\nu} \frac{n_{F}(b \cdot p)\left(1-n_{F}(b \cdot p)\right)}{n_{F}(b \cdot p)},
$$

where $n_{F}$ is the Fermi-Dirac distribution function. This formula is in full agreement with the local equilibrium expression found in ref. [49]. We leave the study of the full resummation of the series (9.6) to future work.

\section{Summary}

In summary, we have derived a general exact form of the Wigner function and the thermal expectation values of local operators of the free Dirac field in the most general case of global thermodynamic equilibrium in Minkowski space-time, that is with a Killing fourtemperature vector including rotation and acceleration. Our method is an extension of that used for the scalar field in ref. [1]: a factorization of the density operator (1.1) and the derivation of the thermal expectation values of quadratic combinations of creation and annihilation operators by iteration. For the spin $1 / 2$ particles, we have obtained the general form of the Wigner function of a free Dirac field as a formal series for imaginary thermal vorticity including all quantum corrections to the classical term. The analytic continuation to real thermal vorticity and the extraction of finite results, demands the application of the analytic distillation, an operation on complex functions introduced in ref. [1] and extended here to the alternate fermionic series. We find that, in the pure acceleration massless case, the method of analytic distillation leads to expressions which all vanish at the Unruh temperature, in agreement with previous findings. Similarly to the scalar field case, analytic distillation defines a new class of complex polynomials which all vanish at $z=2 \pi i$.

We have studied the series in two major cases of non-trivial equilibrium, the pure acceleration and the pure rotation and compared with known perturbative and exact results obtained solving Dirac equation in rotating coordinates. A new exact result has been obtained, namely the thermal expectation value of the axial current and stress-energy tensor in the case of global equilibrium with both acceleration and rotation for the massless case. We have derived the exact expression of the distribution function for massless fermions at global thermodynamic equilibrium, which is an important result for the chiral kinetic theory.

Finally, we derived the exact form of the spin density matrix and the spin polarization vector for the spin $1 / 2$ particles at global thermodynamic equilibrium as a function of thermal vorticity. This latter result is applicable as an improved approximation, with respect to (9.7), to situations where the fields are quasi-free, for instance the final state baryonic effective fields in relativistic heavy ion collisions after hadronization.

\section{Acknowledgments}

M.B. is supported by the Florence University fellowship "Effetti quantistici nei fluidi relativistici". 


\section{A Spinors and group theory}

We review the spinor formalism from a group theory viewpoint. Here, we will focus on the spin $1 / 2$ case, but the formalism can be extended to any spin $[50,51]$. As it is well known, the Dirac field describes a particle (as well as an antiparticle) of spin $1 / 2$, and it transforms in the $(0,1 / 2) \oplus(1 / 2,0)$ projective representation of the ortochronous Lorentz group $\mathrm{SO}(1,3)^{\uparrow}$.

The one-to-one correspondence between four-vectors and $2 \times 2$ hermitian matrices is defined through:

$$
x=x^{\mu} \sigma_{\mu}=x^{0} \mathbb{I}+x^{1} \sigma_{1}+x^{2} \sigma_{2}+x^{3} \sigma_{3}=\left(\begin{array}{cc}
x^{0}+x^{3} & x^{1}-i x^{2} \\
x^{1}+i x^{2} & x^{0}-x^{3}
\end{array}\right) .
$$

where $\sigma_{\mu}=\left(\mathbb{I}, \sigma_{1}, \sigma_{2}, \sigma_{3}\right)$ and $\sigma_{i}$ are the Pauli matrices; also the notation $\bar{\sigma}_{\mu}=(\mathbb{I},-\boldsymbol{\sigma})$ will be used. The Lorentz transformations $\Lambda$ are represented by a $\operatorname{SL}(2, \mathbb{C})$ matrix $D(\Lambda)$ :

$$
\Lambda x=D(\Lambda) x D(\Lambda)^{\dagger}
$$

The complex matrix $D(\Lambda)$ is determined up to a sign, so that the $\operatorname{SL}(2, \mathbb{C}) \leftrightarrow \operatorname{SO}(1,3)^{\uparrow}$ is a 2 to 1 correspondence. The above definition identifies the $(0,1 / 2)$ projective representation $D^{(0,1 / 2)}$ of $\mathrm{SO}(1,3)^{\uparrow}$, where the generators of boosts and rotations are respectively $D^{(0,1 / 2)}\left(K_{i}\right)=i \sigma_{i} / 2$ and $D^{(0,1 / 2)}\left(J_{i}\right)=\sigma_{i} / 2$. Therefore:

$$
D^{(0,1 / 2)}(\Lambda)=D(\Lambda)
$$

We also define the following map:

$$
\widehat{x} \equiv x^{\mu} \bar{\sigma}_{\mu}=x^{0} \mathbb{I}-x^{1} \sigma_{1}-x^{2} \sigma_{2}-x^{3} \sigma_{3}=\left(\begin{array}{cc}
x^{0}-x^{3} & -x^{1}+i x^{2} \\
-x^{1}-i x^{2} & x^{0}+x^{3}
\end{array}\right) .
$$

It is easy to show that:

$$
\widehat{x} \underbrace{x}=x=x \cdot x \mathbb{I}=x^{2} \mathbb{I},
$$

Since (A.1) is a Lorentz invariant, we can infer the transformation law of $\widehat{x}$ from that of $x$. For the eq. (A.1) to be valid in any frame, one needs:

$$
\widehat{\Lambda x}=D(\Lambda)^{\dagger-1} \widehat{x} D(\Lambda)^{-1} .
$$

This map actually corresponds to the $(1 / 2,0)$ representation of $\mathrm{SO}(1,3)^{\uparrow}$, whose generators are $D^{(1 / 2,0)}\left(K_{i}\right)=-i \sigma_{i} / 2$ and $D^{(1 / 2,0)}\left(J_{i}\right)=\sigma_{i} / 2$. Hence:

$$
D^{(1 / 2,0)}(\Lambda)=D^{(0,1 / 2)}(\Lambda)^{\dagger-1}=D(\Lambda)^{\dagger-1}
$$

The quantum single particles states of momentum $p$ in the Hilbert space are usually defined starting from a standard four-momentum $\mathfrak{p}$, and using a standard Lorentz transformation $[p]$ (depending on $p$ and $\mathfrak{p}$ ) such that $\mathfrak{p} \mapsto p$. In formulae:

$$
D([p]) \underbrace{\mathfrak{p} D} D([p])^{\dagger}=\underbrace{p}, \quad D([p])^{\dagger-1} \widehat{\mathfrak{p}} D([p])^{-1}=\widehat{p} .
$$


Also notice, from (A.1), $p \widehat{p}=m^{2} \mathbb{I}$. The choice of $\mathfrak{p}$, which is in principle arbitrary, requires a separate handling of massive and massless particles. Indeed, for massive particles $\mathfrak{p}=(m, 0)$ is basically the only option, whereas in the massless case one usually chooses $\mathfrak{p}=(\kappa, 0,0, \kappa)$ for some positive energy $\kappa$. A crucial role in the Lorentz transformation rules of creation and annihilation operators, and therefore of the field, is played by the so-called little group of $\mathfrak{p}$, that is the group of transformations leaving $\mathfrak{p}$ invariant. In the case of massive particles with $\mathfrak{p}=(m, 0)$, we have $\widehat{\mathfrak{p}}=\underbrace{\mathfrak{p}}=m \mathbb{I}$. Requiring the invariance of these matrices in the equations (A.2), it turns out that the little group is the rotation group $\mathrm{SO}(3)$. In the case of massless particles, choosing the standard vector as $\mathfrak{p}=(\kappa, 0,0, \kappa)$, we have:

$$
\underline{p}=\left(\begin{array}{cc}
2 \kappa & 0 \\
0 & 0
\end{array}\right), \quad \underline{p}=\left(\begin{array}{cc}
0 & 0 \\
0 & 2 \kappa
\end{array}\right),
$$

and the transformations of the little group must have the form:

$$
D([\mathfrak{p}])=\left(\begin{array}{cc}
\mathrm{e}^{-i \phi / 2} & Z \mathrm{e}^{-i \phi / 2} \\
0 & \mathrm{e}^{i \phi / 2}
\end{array}\right)
$$

The parameter $\phi$ is associated with a rotation around the $z$ axis and $Z$ (a complex number) corresponds to a translation in the Euclidean plane [29].

It can be realized that $W(\Lambda, p)=[\Lambda p]^{-1} \Lambda[p]$ belongs to the little group of $\mathfrak{p}$ because it maps $\mathfrak{p}$ to $p$, then to $\Lambda p$ and finally back to $\mathfrak{p}$. The $W(\Lambda, p)$, usually known as the Wigner rotation, dictates how creation and annihilation operators transform under Lorentz transformations represented in the Hilbert space:

$$
\widehat{\Lambda} \widehat{a}_{r}(p) \widehat{\Lambda}^{-1}=\sum_{s} D(W(\Lambda, p))_{r s}^{\dagger} \widehat{a}_{s}(\Lambda p) .
$$

As remarked above, if the particle is massive, $W(\Lambda, p)$ is a rotation, hence $D(W(\Lambda, p))$ is a unitary matrix. In the massless case, it is generally non-unitary, unless the translation parameter $Z$ is set to zero. This is precisely the feature of actual physical representations: known massless particles states transform in representations with $Z=0$ [28]. Therefore, altogether, the little group in both massive and massless cases always acts on physical states with unitary transformations, i.e. $D(W)^{\dagger}=D(W)^{-1}$ in (A.3).

The field in eq. (2.3) with the spinors $(2.4)$ transforms as the $(0,1 / 2) \oplus(1 / 2,0)$ representation of the Lorentz group:

$$
\widehat{\Lambda} \Psi(x) \widehat{\Lambda}^{-1}=S(\Lambda)^{-1} \Psi(\Lambda x),
$$

where $S(\Lambda)$ is given in the eq. (2.5). We show this for the particle term only:

$$
\Psi_{+}(x)=\frac{1}{(2 \pi)^{\frac{3}{2}}} \int \frac{\mathrm{d}^{3} \mathrm{p}}{2 \varepsilon} \mathrm{e}^{-i p \cdot x} \mathrm{U}(p) \widehat{A}(p) .
$$

Making use of the transformation rule (A.3), which in compact notation reads:

$$
\widehat{\Lambda} \widehat{A}(p) \widehat{\Lambda}^{-1}=D(W(\Lambda, p))^{\dagger} \widehat{A}(\Lambda p),
$$


the transformation of the field is:

$$
\widehat{\Lambda} \Psi_{+}(x) \widehat{\Lambda}^{-1}=\frac{1}{(2 \pi)^{\frac{3}{2}}} \int \frac{\mathrm{d}^{3} \mathrm{p}}{2 \varepsilon} \mathrm{e}^{-i p \cdot x} \mathrm{U}(p) D(W(\Lambda, p))^{\dagger} \widehat{A}(\Lambda p) .
$$

Plugging the spinor as in (2.4) and making use of the invariance of $\mathfrak{p}$ under $D(W(\Lambda, p))^{\dagger}=$ $D(W(\Lambda, p))^{-1}=[p]^{-1} \Lambda^{-1}[\Lambda p]$, as well as the transformation rules (A.2), we have:

$$
\begin{aligned}
& \mathrm{U}(p) D(W(\Lambda, p))^{\dagger}=\left(\begin{array}{cc}
D([p]) & 0 \\
0 & D([p])^{\dagger-1}
\end{array}\right)\left(\begin{array}{c}
\underset{\mathcal{p} D(W(\Lambda, p))^{\dagger}}{\widehat{\mathfrak{p}} D(W(\Lambda, p))^{-1}}
\end{array}\right) \\
& =\left(\begin{array}{c}
D([p]) D\left([p]^{-1} \Lambda^{-1}[\Lambda p]\right) \underbrace{\mathfrak{p}} \\
D\left([p]^{\dagger-1}\right) D\left([p]^{\dagger} \Lambda^{\dagger}[\Lambda p]^{\dagger-1}\right) \widehat{\mathfrak{p}}
\end{array}\right) \\
& =\left(\begin{array}{cc}
D\left(\Lambda^{-1}\right) & 0 \\
0 & D\left(\Lambda^{-1}\right)^{\dagger-1}
\end{array}\right) \mathrm{U}(\Lambda p) \\
& =S(\Lambda)^{-1} \mathrm{U}(\Lambda p) \text {. }
\end{aligned}
$$

Finally, after changing the integration variable from $p$ to $\Lambda p$, we obtain:

$$
\widehat{\Lambda} \Psi_{+}(x) \widehat{\Lambda}^{-1}=S(\Lambda)^{-1} \Psi_{+}(\Lambda x) ;
$$

for the antiparticle term the proof is similar. The generators of $S(\Lambda)$ can be written in the form:

$$
\Sigma^{\mu \nu}=\left(\begin{array}{cc}
D^{(0,1 / 2)}\left(J^{\mu \nu}\right) & 0 \\
0 & D^{(1 / 2,0)}\left(J^{\mu \nu}\right)
\end{array}\right) .
$$

By using their form in terms of Pauli matrices, it can be readily checked that they coincide with the better known form $(i / 4)\left[\gamma^{\mu}, \gamma^{\nu}\right]$ where $\gamma$ are in the so-called Weyl representation (2.7).

It is also useful to show that the spinors in eq. (2.4) fulfill the Dirac equation. For the standard momentum $\mathfrak{p}$, we have:

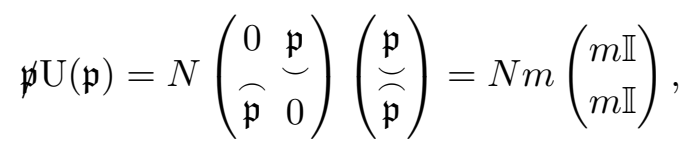

with $N$ normalization factor. Since in the massive case $\mathfrak{p}=\widehat{\mathfrak{p}}=m \mathbb{I}$, and in the massless case $\widehat{\mathfrak{p}} \mathfrak{p}=m^{2}=0$, it is seen that the Dirac equation is satisfied for $p=\mathfrak{p}$. From the transformation rules of the spinors:

$$
\mathrm{U}(p) D(W(\Lambda, p))^{\dagger}=S(\Lambda)^{-1} \mathrm{U}(\Lambda p)
$$

it follows that the equation is fulfilled for any $p$ obtained from $\mathfrak{p}$ by means of a Lorentz transformation. Similarly, one can show that the $V(p)$ spinors fulfill Dirac equation.

The spinors can be written by choosing a particular standard transformations. For instance, in the massive case, the standard transformation can be chosen as the pure 
boost transforming $\mathfrak{p}$ to $p$. This transformation can be written in the $(0,1 / 2)$ representation as [29]:

$$
D([p])=\frac{m+\underline{p}}{\sqrt{2 m(\varepsilon+m)}}
$$

whence the particle spinor in (2.4) follows:

$$
\mathrm{U}(p)=\frac{1}{\sqrt{2 m(\varepsilon+m)}}\left(\begin{array}{cc}
m+\underbrace{p}_{\overparen{p}} & 0 \\
0 & m+\widehat{p}
\end{array}\right) \frac{1}{\sqrt{2 m}}\left(\begin{array}{c}
m \mathbb{I} \\
m \mathbb{I}
\end{array}\right)=\frac{m+\not p}{\sqrt{2 m(\varepsilon+m)}} \mathrm{U}(\mathfrak{p}) .
$$

For massless particles, the standard transformation can be chosen as the boost along the $z$ axis followed by the rotation of axis $\mathbf{k} \times \mathbf{p}$ mapping the $z$ axis to the direction of $\mathbf{p}$. Though more involved, it is then possible to show that [30]:

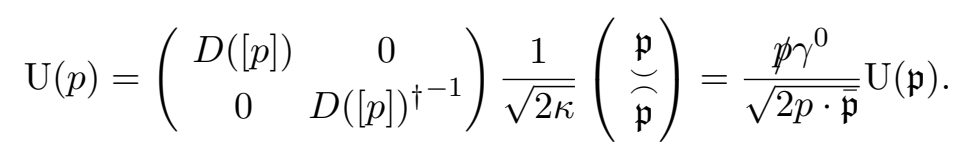

\section{A.1 Massless spinor product}

Taking advantage of the above formulae, it is possible to calculate a useful expression, the spinor product $\bar{u}_{\lambda}\left(p^{\prime}\right) \gamma^{\mu} u_{\lambda}(p)$ for massless particles. To begin with, spinors are written in terms of the standard spinor via the eq. (A.4):

$$
\bar{u}_{\lambda}\left(p^{\prime}\right) \gamma^{\mu} u_{\lambda}(p)=\frac{1}{2 \sqrt{(p \cdot \overline{\mathfrak{p}})\left(p^{\prime} \cdot \overline{\mathfrak{p}}\right)}} \bar{u}_{\lambda}(\mathfrak{p}) \gamma^{0} \not p^{\prime \prime} \gamma^{\mu} \not p \gamma^{0} u_{\lambda}(\mathfrak{p})
$$

The product of matrices in the middle of the right hand side can be written in two ways:

$$
\not p^{\prime \prime} \gamma^{\mu} \not p=\left\{\begin{array}{l}
2 p^{\prime \mu} \not p-\gamma^{\mu} \not p^{\prime \prime} \not p \\
2 p^{\mu} \not p^{\prime \prime}-\not p^{\prime \prime} \not p \gamma^{\mu}
\end{array}=\left\{\begin{array}{l}
2 p^{\prime \mu} \not p-\gamma^{\mu} p \cdot p^{\prime}+2 i \gamma^{\mu} p^{\rho} p^{\sigma} \Sigma_{\rho \sigma} \\
2 p^{\mu} \not p^{\prime \prime}-\gamma^{\mu} p \cdot p^{\prime}+2 i p^{\prime \rho} p^{\sigma} \Sigma_{\rho \sigma} \gamma^{\mu}
\end{array},\right.\right.
$$

where we used the anticommutation rules of the $\gamma$ matrices and the known expression for the product $\not p p p^{\prime \prime}$. Taking the half-sum of the two:

$$
\bar{u}_{\lambda}\left(p^{\prime}\right) \gamma^{\mu} u_{\lambda}(p)=\frac{\bar{u}_{\lambda}(\mathfrak{p}) \gamma^{0}\left(2 p^{\mu} \not p^{\prime \prime}+2 p^{\prime \mu} \not p-2 \gamma^{\mu} p \cdot p^{\prime}+2 i p^{\prime} \rho p^{\sigma}\left\{\Sigma_{\rho \sigma}, \gamma^{\mu}\right\}\right) \gamma^{0} u_{\lambda}(\mathfrak{p})}{4 \sqrt{(p \cdot \overline{\mathfrak{p}})\left(p^{\prime} \cdot \overline{\mathfrak{p}}\right)}} .
$$

This equation can be simplified by using the known relation:

$$
\left\{\Sigma^{\rho \sigma}, \gamma^{\mu}\right\}=-\epsilon^{\mu \rho \sigma \tau} \gamma_{\tau} \gamma_{5}
$$

as well as $\gamma_{5} u_{\lambda}(p)=2 \lambda u_{\lambda}(p)$. Thereby, all terms in the equation (A.5) involve the calculation of the product:

$$
\bar{u}_{\lambda}(\mathfrak{p}) \gamma^{0} \gamma^{\alpha} \gamma^{0} u_{\lambda}(\mathfrak{p})
$$

With $\gamma^{0} \gamma^{0} \gamma^{0}=\gamma^{0}$ and $\gamma^{0} \gamma^{i} \gamma^{0}=-\gamma^{i}$, as well as $\bar{u}_{\lambda}(p) \gamma^{\alpha} u_{\lambda}(p)=2 p^{\alpha}$, this yields:

$$
\bar{u}_{\lambda}(\mathfrak{p}) \gamma^{0} \gamma^{\alpha} \gamma^{0} u_{\lambda}(\mathfrak{p})=2 \overline{\mathfrak{p}}^{\alpha},
$$

where the bar over a vector implies space reflection, i.e. $\bar{p}=\left(p^{0},-\boldsymbol{p}\right)$. By using the above expression into the (A.5), we finally obtain:

$$
\bar{u}_{\lambda}\left(p^{\prime}\right) \gamma^{\mu} u_{\lambda}(p)=\frac{1}{\sqrt{(p \cdot \overline{\mathfrak{p}})\left(p^{\prime} \cdot \overline{\mathfrak{p}}\right)}}\left(p^{\mu} \overline{\mathfrak{p}} \cdot p^{\prime}+p^{\prime \mu} p \cdot \overline{\mathfrak{p}}-\overline{\mathfrak{p}}^{\mu} p \cdot p^{\prime}+2 i \lambda p_{\rho}^{\prime} p_{\sigma} \overline{\mathfrak{p}}_{\tau} \epsilon^{\mu \rho \sigma \tau}\right) .
$$




\section{B Massless fermions and Unruh effect}

In this section we show that the TEVs at global equilibrium with pure acceleration (see subsection 7.1) of a large class of local operators quadratic in the field, vanish at the Unruh temperature $T_{0}=a / 2 \pi$, just like for the scalar field [1]. We show it specifically for the massless Dirac field. The TEVs of local operators quadratic in the field are given by an integral of the Wigner function, just like the operators in eqs. (3.5a), (3.5b) and (3.5c). Hence, by using the exact form of the Wigner function (4.10) they are given by momentum integrals of this sort:

$$
\int \frac{\mathrm{d}^{3} \mathrm{p}}{\varepsilon} p^{\mu_{1}} \cdots p^{\mu_{N}} \partial^{\nu_{1}} \cdots \partial^{\nu_{M}} \mathrm{e}^{-n \tilde{\beta}_{n} \cdot p}=\partial^{\nu_{1}} \cdots \partial^{\nu_{M}} \frac{\partial}{\partial \tilde{\beta}_{n}^{\mu_{1}}} \cdots \frac{\partial}{\partial \tilde{\beta}_{n}^{\mu_{N}}} \frac{(-1)^{N}}{n^{N}} \int \frac{\mathrm{d}^{3} \mathrm{p}}{\varepsilon} \mathrm{e}^{-n \tilde{\beta}_{n} \cdot p} .
$$

These integrals ought to be evaluated for imaginary acceleraration (7.2), and then, after analytic distillation, they can be analytically continued.

As dicussed in subsection 7.1, it is indeed sufficient to calculate the integrals (B.1) in $x=0$. By using the eq. (5.8) and the four-temperature (7.2), it can be seen that integrals such as (B.1), evaluated in $x=0$, result in linear combinations of the following series:

$$
S_{F, 2 m+2}(\phi)=\sum_{n=1}^{\infty}(-1)^{n+1} \frac{\phi^{2 m+2} \sinh (n \phi)}{\sinh ^{2 m+3}(n \phi / 2)} .
$$

Since $S_{F, 2 m+2}$ is a uniformly convergent series of analytic functions for $\operatorname{Re} \phi \neq 0$, it defines an analytic function therein. However, the series is badly divergent for purely imaginary $\phi$ and we need to carry out its analytic distillation at $\phi=0$ in order to obtain a finite physical thermal expectation value. For this purpose we write the series introducing a function $F$ defined by:

$$
S_{F, 2 m+2}(\phi)=\phi^{2 m+2} \sum_{n=1}^{\infty}(-1)^{n+1} F(n \phi) .
$$

In this form we can apply the theorem 1 to obtain an asymptotic series for $S_{F, 2 m+2}$ once we know an asymptotic power expansion about $\phi=0$ of the function $F$. By making use of the generalized version of the Bernoulli polynomials $B_{n}^{(m)}(t)$ defined by [52]:

$$
\left(\frac{x}{\mathrm{e}^{x}-1}\right)^{m} \mathrm{e}^{t x}=\sum_{n=0}^{\infty} \frac{B_{n}^{(m)}(t)}{n !} x^{n},
$$

the function

$$
F(\phi)=\frac{\sinh (\phi)}{\sinh ^{2 m+3}(\phi / 2)}=\frac{2^{2 m+2}}{\phi^{2 m+3}}\left(\frac{\phi}{\mathrm{e}^{\phi}-1}\right)^{2 m+3}\left(\mathrm{e}^{\phi}-\mathrm{e}^{-\phi}\right) \mathrm{e}^{\frac{2 m+3}{2}}
$$

can be written as a convergent power series about $\phi=0$ :

$$
\begin{aligned}
F(\phi) & =2^{2 m+2} \sum_{k=0}^{\infty} \frac{B_{k}^{(2 m+3)}(m+5 / 2)-B_{k}^{(2 m+3)}(m+1 / 2)}{k !} \phi^{k-2 m-3} \\
& =-2^{2 m+3} \sum_{k=0}^{\infty} \frac{B_{2 k+1}^{(2 m+3)}(m+1 / 2)}{(2 k+1) !} \phi^{2 k-2 m-2},
\end{aligned}
$$


where in the last step we used the identities:

$$
\begin{aligned}
& B_{2 k+1}^{(2 m+3)}(m+5 / 2)+B_{2 k+1}^{(2 m+3)}(m+1 / 2)=0, \\
& B_{2 k}^{(2 m+3)}(m+5 / 2)-B_{2 k}^{(2 m+3)}(m+1 / 2)=0,
\end{aligned}
$$

following from the parity of $F(\phi)$ and of the function $\operatorname{coth}(\phi) F(\phi)$. The theorem 1 can be now applied and we obtain:

$$
S_{F, 2 m+2}(\phi) \sim-2^{2 m+3} \sum_{k=0}^{m+1} \frac{B_{2 k+1}^{(2 m+3)}(m+1 / 2)}{(2 k+1) !} \phi^{2 k}\left(1-2^{1+2 k-2 m-2}\right) \zeta(2+2 m-2 k) .
$$

By using the identity

$$
\zeta(2 n)=\frac{(-1)^{n+1} B_{2 n}(2 \pi)^{2 n}}{2(2 n) !}=-\frac{(2 \pi i)^{2 n} B_{2 n}}{2(2 n) !},
$$

where $B_{2 n}$ are Bernoulli numbers, the asymptotic expansion (B.2) can be rewritten as:

$$
\begin{aligned}
S_{F, 2 m+2}(\phi) & \sim-2^{2 m+2} \sum_{k=0}^{m+1}(2 \pi i)^{2 m+2-2 k} \frac{B_{2 m+2-2 k}}{(2+2 m-2 k) !} \frac{B_{2 k+1}^{(2 m+3)}\left(m+\frac{1}{2}\right)}{(2 k+1) !}\left(2^{1+2 k-2 m-2}-1\right) \phi^{2 k} \\
& =-2^{2 m+2} \sum_{k=0}^{m+1}(2 \pi i)^{2 k} \frac{B_{2 k}}{(2 k) !} \frac{B_{2 m+3-2 k}^{(2 m+3)}\left(m+\frac{1}{2}\right)}{(2 m+3-2 k) !}\left(2^{1-2 k}-1\right) \phi^{2 m+2-2 k} .
\end{aligned}
$$

The generalized Bernoulli polynomials fulfill the following identity (see proof below):

$$
\sum_{k=0}^{m+1} \frac{B_{2 k}}{(2 k) !} \frac{B_{2 m+3-2 k}^{(2 m+3)}\left(m+\frac{1}{2}\right)}{(2 m+3-2 k) !}\left(2^{1-2 k}-1\right)=0 .
$$

Using the above identity, after simple calculations, the equation (B.3) can be recast as:

$$
S_{F, 2 m+2}(\phi) \sim 2^{2 m+2} \sum_{k=0}^{m} \frac{B_{2 k+2}}{(2 k+2) !} \frac{B_{2 m+1-2 k}^{(2 m+3)}\left(m+\frac{1}{2}\right)}{(2 m+1-2 k) !}\left(2^{-1-2 k}-1\right) \phi^{2 m-2 k}\left(\phi^{2 k+2}-(2 \pi i)^{2 k+2}\right) .
$$

Finally, taking into account the relation between the value of Bernoulli polynomials and the Bernoulli numbers:

$$
B_{2 k}\left(\frac{1}{2}\right)=\left(2^{1-2 k}-1\right) B_{2 k},
$$

we are in a position to write down the analytic distillation of the series $S_{F, 2 m+2}$ in the following form:

$$
\begin{aligned}
\operatorname{dist}_{0} S_{F, 2 m+2}(\phi) & =-2^{2 m+2} \sum_{k=0}^{m+1}(2 \pi i)^{2 k} \frac{B_{2 k}\left(\frac{1}{2}\right)}{(2 k) !} \frac{B_{2 m+3-2 k}^{(2 m+3)}\left(m+\frac{1}{2}\right)}{(2 m+3-2 k) !} \phi^{2 m+2-2 k} \\
& =2^{2 m+2} \sum_{k=0}^{m} \frac{B_{2 k+2}\left(\frac{1}{2}\right)}{(2 k+2) !} \frac{B_{2 m+1-2 k}^{(2 m+3)}(m+1 / 2)}{(2 m+1-2 k) !} \phi^{2 m-2 k}\left(\phi^{2 k+2}-(2 \pi i)^{2 k+2}\right) .
\end{aligned}
$$

The physical values are obtained by setting $\phi=i a / T_{0}$. As the right hand side of manifestly shows, the polynomials in eq. (B.5) vanish for $T_{0}=a / 2 \pi$, which is just the Unruh temperature. 


\section{B.1 Proof of the equation (B.4)}

We first show that for any integer $M \geq 0$ :

$$
B_{2 M+1}^{(2 M+2)}(M)=0 .
$$

From the Euler Gamma ratio representation [53], with $z \in \mathbb{C}$ and two integers $\alpha, \beta \geq 0$, we have

$$
\frac{\Gamma(z+\alpha)}{\Gamma(z-\beta)}=\sum_{l=0}^{\alpha+\beta} \frac{(\alpha+\beta) !}{l !} \frac{B_{\alpha+\beta-l}^{(1+\alpha+\beta)}(\alpha)}{(\alpha+\beta-l) !} z^{l} .
$$

Then for $M \geq 0$, setting $\alpha=M$ and $\beta=M+1$, we have from (B.7)

$$
\frac{\Gamma(z+M)}{\Gamma(z-M-1)}=\sum_{l=0}^{2 M+1} \frac{(2 M+1) !}{l !(2 M+1-l) !} B_{2 M+1-l}^{(2 M+2)}(M) z^{l} .
$$

At $z=0$, the ratio of Gamma functions in the above expression vanishes because:

$$
\frac{\Gamma(M)}{\Gamma(-M-1)}=\Gamma(M) \Gamma(M+1) \frac{\sin (M \pi)}{\pi}=0 .
$$

However, for $z=0$ in (B.7) the series contains only one term:

$$
\frac{\Gamma(M)}{\Gamma(-M-1)}=B_{2 M+1}^{(2 M+2)}(M)=0,
$$

which proves the equation (B.6).

We are now in a position to prove the identity (B.4). Consider the following series, which follows from the generating function of the Bernoulli numbers:

$$
\left(\frac{x}{\mathrm{e}^{x}-1}+\frac{x}{2}\right)=\sum_{j=0}^{\infty} \frac{B_{2 j}}{(2 j) !} x^{2 j}
$$

and

$$
2\left(\frac{x / 2}{\mathrm{e}^{x / 2}-1}+\frac{x}{4}\right)=\sum_{j=0}^{\infty} \frac{B_{2 j}}{(2 j) !} 2^{1-2 j} x^{2 j} .
$$

Subtracting them, we obtain:

$$
\sum_{j=0}^{\infty} \frac{B_{2 j}}{(2 j) !}\left(2^{1-2 j}-1\right) x^{2 j}=2\left(\frac{x / 2}{\mathrm{e}^{x / 2}-1}+\frac{x}{4}\right)-\left(\frac{x}{\mathrm{e}^{x}-1}+\frac{x}{2}\right)=\frac{x \mathrm{e}^{\frac{x}{2}}}{\mathrm{e}^{x}-1} .
$$

We also need the series:

$$
\sum_{j=0}^{\infty} \frac{B_{j}^{(2 m+3)}\left(m+\frac{1}{2}\right)}{j !} x^{j}=\left(\frac{x \mathrm{e}^{\frac{x}{2}}}{\mathrm{e}^{x}-1}\right)^{2 m+3} \mathrm{e}^{-x} .
$$

The function:

$$
h(x)=\left(\frac{x \mathrm{e}^{\frac{x}{2}}}{\mathrm{e}^{x}-1}\right)\left(\frac{x \mathrm{e}^{\frac{x}{2}}}{\mathrm{e}^{x}-1}\right)^{2 m+3} \mathrm{e}^{-x}=\left(\frac{x \mathrm{e}^{\frac{x}{2}}}{\mathrm{e}^{x}-1}\right)^{2 m+4} \mathrm{e}^{-x},
$$


has the following power series representation:

$$
h(x)=\sum_{n=0}^{\infty} a_{n} x^{n}=\sum_{n=0}^{\infty} \frac{B_{n}^{(2 m+4)}(m+1)}{n !} x^{n} .
$$

It also possible to find another series representation of the function $h$ by making the Cauchy product of the series in (B.8) and (B.9):

$$
\begin{aligned}
h(x) & =\left[\frac{x \mathrm{e}^{\frac{x}{2}}}{\mathrm{e}^{x}-1}\right]\left[\left(\frac{x \mathrm{e}^{\frac{x}{2}}}{\mathrm{e}^{x}-1}\right)^{2 m+3} \mathrm{e}^{-x}\right] \\
& =\left[\sum_{j=0}^{\infty} \frac{B_{2 j}}{(2 j) !}\left(2^{1-2 j}-1\right) x^{2 j}\right]\left[\sum_{j=0}^{\infty} \frac{B_{j}^{(2 m+3)}\left(m+\frac{1}{2}\right)}{j !} x^{j}\right] \\
& =\sum_{k=0}^{\infty} \sum_{l=0}^{k} \frac{B_{2 l}}{(2 l) !}\left(2^{1-2 l}-1\right) \frac{B_{k+l-2 l}^{(2 m+3)}\left(m+\frac{1}{2}\right)}{(k+l-2 l) !} x^{k+l} .
\end{aligned}
$$

Comparing the (B.11) with the (B.10), it can be seen that the $(2 m+3)$-th coefficient of the (B.10) can be retrieved from the (B.11) by setting $k=2 m+3-l$ and summing $l$ from 0 to $m+1$ :

$$
a_{2 m+3}=\frac{B_{2 m+3}^{(2 m+4)}(m+1)}{(2 m+3) !}=\sum_{l=0}^{m+1} \frac{B_{2 l}}{(2 l) !}\left(2^{1-2 l}-1\right) \frac{B_{2 m+3-2 l}^{(2 m+3)}\left(m+\frac{1}{2}\right)}{(2 m+3-2 l) !} .
$$

Taking into account the eq. (B.6) with $M=m+1$, it turns out that $a_{2 m+3}$ vanishes, that is:

$$
\sum_{l=0}^{m+1} \frac{B_{2 l}}{(2 l) !}\left(2^{1-2 l}-1\right) \frac{B_{2 m+3-2 l}^{(2 m+3)}\left(m+\frac{1}{2}\right)}{(2 m+3-2 l) !}=0,
$$

which is the identity (B.4) we wanted to prove.

\section{Analytic distillation for pure rotation}

We go through some steps in the calculation of the mean value of the stress-energy tensor and the axial current of the massless Dirac field at global equilibrium with rotation. In this case, the tensors $\Lambda^{n}, S(\Lambda)^{n}, A$ and $A_{5}$ in eq. (5.5) and $A_{5}$ in eq. (5.6) are obtained from the eq. (7.6) and are given in (7.9).

Plugging these expression into the eq. (5.10) and (5.9), one obtains the exact mean value of the canonical stress-energy tensor and of the axial current as a series. The Belinfante stress-energy tensor is then obtained form the canonical one from the simple relation (7.10):

$$
T_{B}^{\mu \nu}(x)_{I}=\lim _{B \rightarrow \phi} \frac{1}{2 \pi^{2}} \sum_{n=1}^{\infty} \frac{(-1)^{n+1}}{\left(n^{2} \phi^{2}+4 B^{2} r^{2} T_{0}^{2} \sin ^{2}\left(\frac{n \phi}{2}\right)\right)^{3}} \Theta_{n}^{\mu \nu}(x),
$$


where the tensor $\Theta_{n}(x)$ has the following components:

$$
\begin{aligned}
& \Theta_{n}^{00}(x)=8 B^{4} T_{0}^{4} \cos \left(\frac{n \phi}{2}\right)\left[3 n^{2} \phi^{2}+2 B^{2} r^{2} T_{0}^{2}(\cos n \phi-1)\right], \\
& \Theta_{n}^{11}(x)=8 B^{4} T_{0}^{4} \cos \left(\frac{n \phi}{2}\right)\left(B^{2} T_{0}^{2}\left(r^{2}-4 y^{2}\right)+n^{2} \phi^{2}\right)-8 B^{6} T_{0}^{6}\left(r^{2}-4 y^{2}\right) \cos \left(\frac{3 n \phi}{2}\right), \\
& \Theta_{n}^{22}(x)=8 B^{4} T_{0}^{4} \cos \left(\frac{n \phi}{2}\right)\left(B^{2} T_{0}^{2}\left(r^{2}-4 x^{2}\right)+n^{2} \phi^{2}\right)-8 B^{6} T_{0}^{6}\left(r^{2}-4 x^{2}\right) \cos \left(\frac{3 n \phi}{2}\right), \\
& \Theta_{n}^{33}(x)=8 B^{4} T_{0}^{4} \cos \left(\frac{n \phi}{2}\right)\left(n^{2} \phi^{2}+4 B^{2} r^{2} T_{0}^{2} \sin ^{2}\left(\frac{n \phi}{2}\right)\right), \\
& \Theta_{n}^{01}(x)=16 i B^{5} T_{0}^{5} y n \phi \sin \left(\frac{n \phi}{2}\right)(\cos (n \phi)+3), \\
& \Theta_{n}^{02}(x)=-16 i B^{5} T_{0}^{5} x n \phi \sin \left(\frac{n \phi}{2}\right)(\cos (n \phi)+3), \\
& \Theta_{n}^{12}(x)=64 B^{6} T_{0}^{6} x y \sin \left(\frac{n \phi}{2}\right) \sin (n \phi) .
\end{aligned}
$$

Note the introduction of the auxiliary real parameter $B$ and the exchange of the limit and series as discussed in subsection 7.2, with $\phi$ and $B$ both real.

The calculation proceeds as outlined in subsection 7.2 : by using theorem 1 , the power asymptotic series in $\phi$ of the $B$-dependent components of the stress-energy tensor are determined; then, the limit $B \rightarrow \phi$ is taken and the resulting expressions implicitly provide the full asymptotic power series in $\phi$ about $\phi=0$ which are needed to determine the analytic distillate. Finally, the vorticity is continued to its physical value via the mapping $\phi \rightarrow i \omega / T_{0}$. Eventually, the following expressions are obtained:

$$
\begin{aligned}
T_{B}^{00}= & \frac{7}{180} \pi^{2}\left(4 \gamma^{2}-1\right) \gamma^{4} T_{0}^{4}+\frac{1}{72}\left(24 \gamma^{4}-16 \gamma^{2}+1\right) \gamma^{4} T_{0}^{2} \omega^{2} \\
& +\frac{\left(960 \gamma^{6}-1128 \gamma^{4}+196 \gamma^{2}+17\right) \gamma^{4} \omega^{4}}{2880 \pi^{2}}, \\
T_{B}^{11}= & \frac{7}{180} \pi^{2} \gamma^{4} T_{0}^{4}+\frac{1}{360} \gamma^{4} T_{0}^{2} \omega^{2}\left(4 \gamma^{2}\left(14 \pi^{2} T_{0}^{2} y^{2}+5\right)-5\right) \\
& +\frac{\gamma^{4} \omega^{4}\left(120 \gamma^{4}\left(8 \pi^{2} T_{0}^{2} y^{2}+1\right)-8 \gamma^{2}\left(20 \pi^{2} T_{0}^{2} y^{2}+11\right)-17\right)}{2880 \pi^{2}} \\
& +\frac{\gamma^{6}\left(240 \gamma^{4}-132 \gamma^{2}-17\right) y^{2} \omega^{6}}{720 \pi^{2}} \\
T_{B}^{22}= & \frac{7}{180} \pi^{2} \gamma^{4} T_{0}^{4}+\frac{1}{360} \gamma^{4} T_{0}^{2} \omega^{2}\left(4 \gamma^{2}\left(14 \pi^{2} T_{0}^{2} x^{2}+5\right)-5\right) \\
& +\frac{\gamma^{4} \omega^{4}\left(120 \gamma^{4}\left(8 \pi^{2} T_{0}^{2} x^{2}+1\right)-8 \gamma^{2}\left(20 \pi^{2} T_{0}^{2} x^{2}+11\right)-17\right)}{2880 \pi^{2}} \\
& +\frac{\gamma^{6}\left(240 \gamma^{4}-132 \gamma^{2}-17\right) x^{2} \omega^{6}}{720 \pi^{2}} \\
T_{B}^{33}= & \frac{7}{180} \pi^{2} \gamma^{4} T_{0}^{4}+\frac{1}{72}\left(4 \gamma^{2}-1\right) \gamma^{4} T_{0}^{2} \omega^{2}+\frac{\left(120 \gamma^{4}-88 \gamma^{2}-17\right) \gamma^{4} \omega^{4}}{2880 \pi^{2}}, \\
T_{B}^{01}= & -\frac{7}{45} \pi^{2} \gamma^{6} T_{0}^{4} y \omega+\frac{1}{9}\left(1-3 \gamma^{2}\right) \gamma^{6} T_{0}^{2} y \omega^{3}-\frac{\left(80 \gamma^{4}-64 \gamma^{2}+15\right) \gamma^{6} y \omega^{5}}{240 \pi^{2}} \\
T_{B}^{02}= & \frac{7}{45} \pi^{2} \gamma^{6} T_{0}^{4} x \omega-\frac{1}{9}\left(1-3 \gamma^{2}\right) \gamma^{6} T_{0}^{2} x \omega^{3}+\frac{\left(80 \gamma^{4}-64 \gamma^{2}+15\right) \gamma^{6} x \omega^{5}}{240 \pi^{2}}, \\
T_{B}^{12}= & -\frac{7}{45} \pi^{2} \gamma^{6} T_{0}^{4} x y \omega^{2}+\frac{1}{18}\left(1-6 \gamma^{2}\right) \gamma^{6} T_{0}^{2} x y \omega^{4}-\frac{\left(240 \gamma^{4}-132 \gamma^{2}-17\right) \gamma^{6} x y \omega^{6}}{720 \pi^{2}},
\end{aligned}
$$


where $\gamma=1 / \sqrt{1-r^{2} \omega^{2}}$. To finally derive the coefficients appearing in the decomposition of eq. (7.14), the relations:

$$
\begin{aligned}
u^{\mu} & =\gamma(1,-y \omega, x \omega, 0), & \alpha^{\mu} & =\left(0,-\frac{\gamma x \omega^{2}}{T_{0}},-\frac{\gamma y \omega^{2}}{T_{0}}, 0\right), \\
w^{\mu} & =\left(0,0,0, \frac{\gamma \omega}{T_{0}}\right), & l^{\mu} & =\left(\frac{\gamma\left(\gamma^{2}-1\right) \omega^{2}}{T_{0}^{2}},-\frac{\gamma^{3} y \omega^{3}}{T_{0}^{2}}, \frac{\gamma^{3} x \omega^{3}}{T_{0}^{2}}, 0\right),
\end{aligned}
$$

and:

$$
\beta^{2}(x)=\frac{1}{T_{0}^{2} \gamma^{2}}, \quad \alpha^{2}(x)=-\left(\gamma^{2}-1\right) \frac{\omega^{2}}{T_{0}^{2}}, \quad w^{2}(x)=-\gamma^{2} \frac{\omega^{2}}{T_{0}^{2}},
$$

must be used. The results are quoted in (7.15).

The mean value of the axial current is obtained likewise. For equilibrium with rotation, the only non-vanishing component of the series (5.9) is directed along the axis of rotation:

$$
j_{A}^{3}(x)_{I}=\lim _{B \rightarrow \phi}-\frac{4 i B^{3} T_{0}^{3}}{\pi^{2}} \sum_{n=1}^{\infty}(-1)^{n+1} \frac{n \phi \sin \left(\frac{n \phi}{2}\right)}{\left(n^{2} \phi^{2}+4 B^{2} T_{0}^{2} r^{2} \sin ^{2}\left(\frac{n \phi}{2}\right)\right)^{2}} .
$$

The series is handled like for the stress-energy tensor components and it yields:

$$
j_{A}^{3}(x)=\left.\operatorname{dist}_{0} j_{A}^{3}(x)_{I}\right|_{\phi=i \omega / T_{0}}=\frac{1}{6} \gamma^{4} T_{0}^{2} \omega+\frac{\gamma^{4} \omega^{3}}{24 \pi^{2}}\left(4 \gamma^{2}-3\right) .
$$

This is the same result obtained in [42]. Employing the tetrad defined above, the axial current can be written in the form of eq. (7.16).

\section{Analytic distillation for rotation and acceleration}

Here we go through some steps of the analytic distillation of the stress-energy tensor and the axial current of the free massless Dirac field when equilibrium includes both a rotation and acceleration along the $z$-axis; the corresponding calculation for the scalar field was carried out in ref. [1]. The series of the canonical/Belinfante stress-energy tensor and the axial current are obtained by using the expressions (7.17) in eqs. (5.10) and (5.9).

In order to apply theorem 1, the series obtained with eq. (5.10) and (5.9) are to be rewritten in a suitable form by introducing auxiliary parameters (thereafter $B, C$ and, when needed $D$ ). Particularly, the Belinfante stress-energy tensor can be written as:

$$
T_{B}^{\mu \nu}(x)_{I}=\lim _{B, C, D \rightarrow \bar{B}, \bar{C}, \bar{D}} \frac{1}{2 \pi^{2}} \sum_{n=1}^{\infty} \frac{(-1)^{n+1}}{2\left(B \sinh ^{2}\left(\frac{n \Phi}{2}\right)+C \sin ^{2}\left(\frac{n \phi}{2}\right)\right)^{3}} \Theta_{n}^{\mu \nu}(x, B, C, D),
$$

where ( $t, z$ being the Cartesian coordinates):

$$
\bar{B}=\Phi^{2} T_{0}^{2} t^{2}+\left(1-i \Phi T_{0} z\right)^{2}, \quad \bar{C}=r^{2} \Phi^{2} T_{0}^{2},
$$

and $\bar{D}$ is a component-dependent quantity:

$$
\bar{D}_{00}=\left(T_{0} \Phi z+i\right), \quad \bar{D}_{11}=T_{0} \Phi y, \quad \bar{D}_{22}=T_{0} \Phi x, \quad \bar{D}_{33}=T_{0} \Phi t .
$$


In the above expressions, limits can be exchanged with the series because they are uniformly convergent series of continuous functions of the arguments $B, C, D$, for real values and for real $\phi$ and $\Phi .{ }^{4}$ The tensor $\Theta$ reads:

$$
\begin{aligned}
& \Theta_{n}^{00}=-T_{0}^{4} \Phi^{4} \cosh \left(\frac{n \Phi}{2}\right) \cos \left(\frac{n \phi}{2}\right)\left(B \sinh ^{2}\left(\frac{n \Phi}{2}\right)+C \sin ^{2}\left(\frac{n \phi}{2}\right)+2 D_{00}^{2}(\cosh (n \Phi)-1)\right), \\
& \Theta_{n}^{11}=T_{0}^{4} \Phi^{4} \cosh \left(\frac{n \Phi}{2}\right) \cos \left(\frac{n \phi}{2}\right)\left(B \sinh ^{2}\left(\frac{n \Phi}{2}\right)+C \sin ^{2}\left(\frac{n \phi}{2}\right)+2 D_{11}^{2}(\cos (n \phi)-1)\right), \\
& \Theta_{n}^{33}=T_{0}^{4} \Phi^{4} \cosh \left(\frac{n \Phi}{2}\right) \cos \left(\frac{n \phi}{2}\right)\left(B \sinh ^{2}\left(\frac{n \Phi}{2}\right)+C \sin ^{2}\left(\frac{n \phi}{2}\right)-2 D_{33}^{2}(\cosh (n \Phi)-1)\right), \\
& \Theta_{n}^{01}=T_{0}^{5} \Phi^{5} y\left(i+T_{0} \Phi z\right) \sinh \left(\frac{n \Phi}{2}\right) \sin \left(\frac{n \phi}{2}\right)(\cosh (n \Phi)+\cos (n \phi)+2), \\
& \Theta_{n}^{03}=-T_{0}^{5} \Phi^{5} t\left(i+T_{0} \Phi z\right) \sinh \left(\frac{n \Phi}{2}\right) \sinh (n \Phi) \sin (n \phi) \csc \left(\frac{n \phi}{2}\right), \\
& \Theta_{n}^{12}=T_{0}^{6} \Phi^{6} x y \sinh (n \Phi) \operatorname{csch}\left(\frac{n \Phi}{2}\right) \sin \left(\frac{n \phi}{2}\right) \sin (n \phi) \\
& \Theta_{n}^{13}=T_{0}^{6} \Phi^{6} t y \sinh \left(\frac{n \Phi}{2}\right) \sin \left(\frac{n \phi}{2}\right)(\cosh (n \Phi)+\cos (n \phi)+2), \\
& \Theta_{n}^{22}=\Theta_{n}^{11}(y \mapsto x), \quad \Theta_{n}^{02}=\Theta_{n}^{01}(y \mapsto-x), \quad \Theta_{n}^{23}=\Theta_{n}^{12}(y \mapsto-x) .
\end{aligned}
$$

As they stand, the components of the stress-energy tensor are proportional to alternatingsign series of functions of $n \phi$ and $n \Phi$. To use the theorem 1, we define the map:

$$
\Phi=\xi \cos \theta \quad \phi=\xi \sin \theta
$$

thereby obtaining series of functions of $n \xi$. The asymptotic expansion of each component of the stress-energy tensor can now be calculated. Proceeding as in the previous cases, i.e. evaluating the limits in the auxiliary parameters, obtaining the analytic distillate and continuing to the physical values of thermal vorticity, we get the exact TEVs. To sketch the various steps, we focus on the time-time component of the Belinfante stress-energy tensor as an example:

$$
\begin{gathered}
T_{B}^{00}(x)_{I}=-\frac{T_{0}^{4} \Phi^{4}}{4 \pi^{2}} \sum_{n=1}^{\infty} \lim _{B, C, D \rightarrow \bar{B}, \bar{C}, \bar{D}}(-1)^{n+1} \cosh \left(\frac{n \Phi}{2}\right) \cos \left(\frac{n \phi}{2}\right) \times \\
\frac{B \sinh ^{2}\left(\frac{n \Phi}{2}\right)+C \sin ^{2}\left(\frac{n \phi}{2}\right)+2(\cosh (n \Phi)-1) D^{2}}{\left(B \sinh ^{2}\left(\frac{n \Phi}{2}\right)+C \sin ^{2}\left(\frac{n \phi}{2}\right)\right)^{3}},
\end{gathered}
$$

where $\bar{B}, \bar{C}, \bar{D}$ like in eq. (D.1)(D.2) ( $D=D_{00}$ and $\bar{D}=\bar{D}_{00}$ are implied). The transformation (D.3) is applied and a series of functions $f(n \xi)$ is thus obtained:

$$
\begin{aligned}
T_{B}^{00}(x)_{I}= & \lim _{B, C, D \rightarrow \bar{B}, \bar{C}, \bar{D}}-\frac{T_{0}^{4} \xi^{4} \cos ^{4} \theta}{4 \pi^{2}} \sum_{n=1}^{\infty} \frac{(-1)^{n+1} \cosh \left(\frac{n \xi \cos \theta}{2}\right) \cos \left(\frac{n \xi \sin \theta}{2}\right)}{\left(B \sinh ^{2}\left(\frac{n \xi \cos \theta}{2}\right)+C \sin ^{2}\left(\frac{n \xi \sin \theta}{2}\right)\right)^{3}} \times \\
& \left(B \sinh ^{2}\left(\frac{n \xi \cos \theta}{2}\right)+C \sin ^{2}\left(\frac{n \xi \sin \theta}{2}\right)+2(\cosh (n \xi \cos \theta)-1) D^{2}\right) \\
\equiv & \lim _{B, C, D \rightarrow \bar{B}, \bar{C}, \bar{D}}-\frac{T_{0}^{4} \xi^{4} \cos ^{4} \theta}{4 \pi^{2}} G(\theta, \xi, B, C, D) .
\end{aligned}
$$

\footnotetext{
${ }^{4}$ Indeed the series does not converge for $B=0$, which is impossible as long as $T_{0}>0$.
} 
The asymptotic power expansion in $\xi$ of the series $G$ can now be obtained by using theorem 1:

$$
\begin{aligned}
G \sim & \frac{7 \pi^{4}\left(\left(B+4 D^{2}\right) c^{2}+C s^{2}\right)}{45 \xi^{4}\left(B c^{2}+C s^{2}\right)^{3}} \\
& -\frac{\pi^{2}}{18 \xi^{2}\left(B c^{2}+C s^{2}\right)^{4}}\left[s^{2} c^{4}\left(3 B^{2}-2 B\left(C-6 D^{2}\right)-20 C D^{2}\right)\right. \\
& \left.+C s^{4} c^{2}\left(2 B-3\left(C+4 D^{2}\right)\right)+B\left(B+4 D^{2}\right) c^{6}-C^{2} s^{6}\right] \\
& +\frac{1}{720\left(B c^{2}+C s^{2}\right)^{5}}\left[B s^{2} c^{8}\left(30 B^{2}-139 B C+120 B D^{2}-664 C D^{2}\right)\right. \\
& +C s^{6} c^{4}\left(15 C\left(C+40 D^{2}\right)-107 B^{2}-6 B\left(25 C+132 D^{2}\right)\right) \\
& -17 B^{2}\left(B+4 D^{2}\right) c^{10}+s^{4} c^{6}\left(15 B^{3}-30 B^{2}\left(5 C-2 D^{2}\right)\right. \\
& \left.-B C\left(107 C+1200 D^{2}\right)+364 C^{2} D^{2}\right) \\
& \left.+C^{2} s^{8} c^{2}\left(-139 B+30 C+108 D^{2}\right)-17 C^{3} s^{10}\right],
\end{aligned}
$$

where

$$
c=\cos \theta, \quad s=\sin \theta .
$$

Having obtained the asymptotic power expansion of $G$ in powers of $\xi$ about $\xi=0$, which we shall denote as $G_{A}$, we can compute the limits in the auxiliary parameters and transform the variables $\xi, \theta$ back to $\Phi, \phi$. Hence:

$$
\operatorname{dist}_{0} T_{B}^{00}(x)(\phi, \Phi)_{I}=\operatorname{dist}_{0} \frac{T_{0}^{4} \Phi^{4}}{4 \pi^{2}} G_{A}(\Phi, \phi, \bar{B}, \bar{C}, \bar{D}) .
$$

Finally, we can set $\Phi \rightarrow i a / T_{0}$ and $\phi \rightarrow i \omega / T_{0}$ to get the physical value by analytic continuation:

$$
T_{B}^{00}(x)=\frac{a^{4}}{4 \pi^{2}} G_{A}\left(i a / T_{0}, i \omega / T_{0}, \bar{B}, \bar{C}, \bar{D}\right),
$$

where the arguments of $\bar{B}, \bar{C}, \bar{D}$ in eqs. (D.1), (D.2) are also continued. The other components can be worked out in a similar fashion. The resulting coefficients of the general decomposition (7.14) are quoted in eq. (7.18). They can be extracted by comparing the various components with the decomposition onto the tetrad:

$$
\begin{aligned}
u^{\mu} & =\gamma(1+a z,-\omega y, \omega x, a t), \\
\alpha^{\mu} & =\frac{\gamma}{T_{0}}\left(a^{2} t,-\omega^{2} x,-\omega^{2} y, a(1+a z)\right), \\
w^{\mu} & =\frac{\gamma \omega}{T_{0}}(a t, a x, a y, 1+a z), \\
l^{\mu} & =\frac{\gamma^{3} \omega\left(a^{2}+\omega^{2}\right)}{T_{0}^{2}}\left(\omega r^{2}(1+a z),-y\left((1+a z)^{2}-a^{2} t^{2}\right), x\left((1+a z)^{2}-a^{2} t^{2}\right), r^{2} a t\right),
\end{aligned}
$$

with:

$$
\begin{aligned}
\beta^{2} & =\frac{a^{2}\left(z^{2}-t^{2}\right)+2 a z-r^{2} \omega^{2}+1}{T_{0}^{2}}, & \alpha^{2} & =-\frac{a^{4}\left(z^{2}-t^{2}\right)+2 a^{3} z+a^{2}+r^{2} \omega^{4}}{T_{0}^{4} \beta^{2}}, \\
w^{2} & =-\frac{\omega^{2}\left(a^{2}\left(r^{2}-t^{2}+z^{2}\right)+2 a z+1\right)}{T_{0}^{4} \beta^{2}}, & \alpha \cdot w & =-a \omega / T_{0}^{2},
\end{aligned}
$$

where $\gamma=\left((1+a z)^{2}-a^{2} t^{2}-\omega^{2} r^{2}\right)^{-1 / 2}$. 
The axial current can be obtained from the series (5.9):

$$
j_{A}^{\mu}(x)_{I}=\lim _{B, C \rightarrow \bar{B}, \bar{C}} \frac{\Phi^{3} T_{0}^{3}}{2 \pi^{2}} \sum_{n=1}^{\infty} \frac{(-1)^{n+1}}{\left(B \sinh \left(\frac{n \Phi}{2}\right)+C \sin \left(\frac{n \phi}{2}\right)\right)^{2}} \Upsilon_{n}^{\mu}
$$

where $\bar{B}$ and $\bar{C}$ are the same as in (D.1), (D.2) and the vector $\Upsilon$ reads:

$$
\begin{aligned}
& \Upsilon^{0}=-\Phi t T_{0} \sin \left(\frac{n \phi}{2}\right) \sinh \left(\frac{n \Phi}{2}\right), \\
& \Upsilon^{3}=-\left(i+\Phi T_{0} z\right) \sin \left(\frac{n \phi}{2}\right) \sinh \left(\frac{n \Phi}{2}\right), \\
& \Upsilon^{1}=\Upsilon^{0}(t \mapsto x), \\
& \Upsilon^{2}=\Upsilon^{0}(t \mapsto y) .
\end{aligned}
$$

The method is the same as for the stress-energy tensor, which eventually yields the covariant expression (7.16), with no extra dependence on $\alpha \cdot w$.

Open Access. This article is distributed under the terms of the Creative Commons Attribution License (CC-BY 4.0), which permits any use, distribution and reproduction in any medium, provided the original author(s) and source are credited.

\section{References}

[1] F. Becattini, M. Buzzegoli and A. Palermo, Exact equilibrium distributions in statistical quantum field theory with rotation and acceleration: scalar field, JHEP 02 (2021) 101 [arXiv : 2007.08249] [INSPIRE].

[2] N. Weickgenannt, X.-L. Sheng, E. Speranza, Q. Wang and D.H. Rischke, Kinetic theory for massive spin-1/2 particles from the Wigner-function formalism, Phys. Rev. D 100 (2019) 056018 [arXiv: 1902.06513] [INSPIRE].

[3] N. Weickgenannt, E. Speranza, X.-l. Sheng, Q. Wang and D.H. Rischke, Generating Spin Polarization from Vorticity through Nonlocal Collisions, Phys. Rev. Lett. 127 (2021) 052301 [arXiv:2005.01506] [INSPIRE].

[4] Z. Wang, X. Guo, S. Shi and P. Zhuang, Mass Correction to Chiral Kinetic Equations, Phys. Rev. D 100 (2019) 014015 [arXiv: 1903. 03461] [INSPIRE].

[5] K. Hattori, Y. Hidaka and D.-L. Yang, Axial Kinetic Theory and Spin Transport for Fermions with Arbitrary Mass, Phys. Rev. D 100 (2019) 096011 [arXiv:1903.01653] [INSPIRE].

[6] J.-H. Gao and Z.-T. Liang, Relativistic Quantum Kinetic Theory for Massive Fermions and Spin Effects, Phys. Rev. D 100 (2019) 056021 [arXiv: 1902.06510] [INSPIRE].

[7] Z. Wang, X. Guo and P. Zhuang, Equilibrium Spin Distribution From Detailed Balance, Eur. Phys. J. C 81 (2021) 799 [arXiv:2009.10930] [InSPIRE].

[8] D.-L. Yang, K. Hattori and Y. Hidaka, Effective quantum kinetic theory for spin transport of fermions with collsional effects, JHEP 07 (2020) 070 [arXiv:2002.02612] [INSPIRE].

[9] F. Becattini and M.A. Lisa, Polarization and Vorticity in the Quark-Gluon Plasma, Ann. Rev. Nucl. Part. Sci. 70 (2020) 395 [arXiv:2003. 03640] [InSPIRE]. 
[10] D.T. Son and N. Yamamoto, Kinetic theory with Berry curvature from quantum field theories, Phys. Rev. D 87 (2013) 085016 [arXiv: 1210.8158] [INSPIRE].

[11] M.A. Stephanov and Y. Yin, Chiral Kinetic Theory, Phys. Rev. Lett. 109 (2012) 162001 [arXiv: 1207.0747] [INSPIRE].

[12] J.-Y. Chen, D.T. Son, M.A. Stephanov, H.-U. Yee and Y. Yin, Lorentz Invariance in Chiral Kinetic Theory, Phys. Rev. Lett. 113 (2014) 182302 [arXiv:1404.5963] [inSPIRE].

[13] Y. Hidaka, S. Pu and D.-L. Yang, Relativistic Chiral Kinetic Theory from Quantum Field Theories, Phys. Rev. D 95 (2017) 091901 [arXiv:1612.04630] [InSPIRE].

[14] J.-h. Gao, S. Pu and Q. Wang, Covariant chiral kinetic equation in the Wigner function approach, Phys. Rev. D 96 (2017) 016002 [arXiv:1704.00244] [INSPIRE].

[15] S. Shi, C. Gale and S. Jeon, From chiral kinetic theory to relativistic viscous spin hydrodynamics, Phys. Rev. C 103 (2021) 044906 [arXiv: 2008.08618] [InSPIRE].

[16] Y.-C. Liu, L.-L. Gao, K. Mameda and X.-G. Huang, Chiral kinetic theory in curved spacetime, Phys. Rev. D 99 (2019) 085014 [arXiv:1812.10127] [InSPIRE].

[17] C. Manuel and J.M. Torres-Rincon, Kinetic theory of chiral relativistic plasmas and energy density of their gauge collective excitations, Phys. Rev. D 89 (2014) 096002 [arXiv: 1312.1158] [INSPIRE].

[18] A. Huang, S. Shi, Y. Jiang, J. Liao and P. Zhuang, Complete and Consistent Chiral Transport from Wigner Function Formalism, Phys. Rev. D 98 (2018) 036010 [arXiv: 1801.03640] [INSPIRE].

[19] J.-W. Chen, S. Pu, Q. Wang and X.-N. Wang, Berry curvature and four-dimensional monopoles in the relativistic chiral kinetic equation, Phys. Rev. Lett. 110 (2013) 262301.

[20] N. Mueller and R. Venugopalan, Chiral anomaly, berry phase and chiral kinetic theory from worldlines in quantum field theory, Phys. Rev. D 97 (2018) 051901.

[21] Y.-C. Liu, K. Mameda and X.-G. Huang, Covariant Spin Kinetic Theory I: Collisionless Limit, Chin. Phys. C 44 (2020) 094101 [Erratum ibid. 45 (2021) 089001] [arXiv: 2002.03753] [INSPIRE].

[22] F. Becattini, Covariant statistical mechanics and the stress-energy tensor, Phys. Rev. Lett. 108 (2012) 244502 [arXiv:1201.5278] [INSPIRE].

[23] F. Becattini and E. Grossi, Quantum corrections to the stress-energy tensor in thermodynamic equilibrium with acceleration, Phys. Rev. D 92 (2015) 045037 [arXiv: 1505. 07760] [INSPIRE].

[24] M. Buzzegoli and F. Becattini, General thermodynamic equilibrium with axial chemical potential for the free Dirac field, JHEP 12 (2018) 002 [arXiv:1807.02071] [INSPIRE].

[25] F. Becattini, L. Bucciantini, E. Grossi and L. Tinti, Local thermodynamical equilibrium and the beta frame for a quantum relativistic fluid, Eur. Phys. J. C 75 (2015) 191 [arXiv: 1403.6265] [INSPIRE].

[26] F. Becattini, M. Buzzegoli and E. Grossi, Reworking the Zubarev's approach to non-equilibrium quantum statistical mechanics, Particles 2 (2019) 197.

[27] S. Weinberg, The Quantum theory of fields. Vol. 1: Foundations, Cambridge University Press (2005) [ISBN: 9780521670531].

[28] W. Tung, Group Theory in Physics, World Scientific (1985) [DOI]. 
[29] P. Moussa and R. Stora, Angular analysis of elementary particle reactions, in Proc. of the 1966 International School on Elementary Particles, Herceg Novi Yugoslavia (1966) [INSPIRE].

[30] S.D. Groot, W. van Leeuwen and C. van Weert, Relativistic Kinetic Theory. Principles and Applications, North Holland, Amsterdam (1980) [InSPIRE].

[31] E. Zeidler, Quantum Field Theory I: Basics in Mathematics and Physics: A Bridge between Mathematicians and Physicists, Springer Berlin Heidelberg (2007) [DOI].

[32] D. Zagier, Appendix. The Mellin transform and related analytic techniques, (2006) [http://people.mpim-bonn.mpg.de/zagier/files/tex/MellinTransform/fulltext.pdf].

[33] F. Becattini, Thermodynamic equilibrium with acceleration and the Unruh effect, Phys. Rev. D 97 (2018) 085013 [arXiv: 1712.08031] [INSPIRE].

[34] M. Buzzegoli, E. Grossi and F. Becattini, General equilibrium second-order hydrodynamic coefficients for free quantum fields, JHEP 10 (2017) 091 [Erratum ibid. 07 (2018) 119] [arXiv: 1704.02808] [INSPIRE].

[35] F. Becattini and D. Rindori, Extensivity, entropy current, area law and Unruh effect, Phys. Rev. D 99 (2019) 125011 [arXiv:1903.05422] [INSPIRE].

[36] G.Y. Prokhorov, O.V. Teryaev and V.I. Zakharov, Unruh effect universality: emergent conical geometry from density operator, JHEP 03 (2020) 137 [arXiv: 1911. 04545] [INSPIRE].

[37] G.Y. Prokhorov, O.V. Teryaev and V.I. Zakharov, Unruh effect for fermions from the Zubarev density operator, Phys. Rev. D 99 (2019) 071901 [arXiv:1903.09697] [InSPIRE].

[38] G.Y. Prokhorov, O.V. Teryaev and V.I. Zakharov, Calculation of acceleration effects using the Zubarev density operator, Particles 3 (2020) 1 [arXiv:1911.04563].

[39] W. Florkowski, E. Speranza and F. Becattini, Perfect-fluid hydrodynamics with constant acceleration along the stream lines and spin polarization, Acta Phys. Polon. B 49 (2018) 1409 [arXiv: 1803.11098] [INSPIRE].

[40] V.E. Ambrus and E. Winstanley, Rotating fermions inside a cylindrical boundary, Phys. Rev. D 93 (2016) 104014 [arXiv: 1512.05239] [INSPIRE].

[41] V.E. Ambruş and E. Winstanley, Rotating quantum states, Phys. Lett. B 734 (2014) 296 [arXiv: 1401.6388] [INSPIRE].

[42] V.E. Ambrus and E. Winstanley, Exact solutions in quantum field theory under rotation, (2019) [arXiv:arXiv: 1908.10244].

[43] V.E. Ambrus, Dirac fermions on rotating space-times, Ph.D. Thesis, Sheffield University, Sheffield, U.K. (2014) [inSPIRE].

[44] G.Y. Prokhorov, O.V. Teryaev and V.I. Zakharov, Effects of rotation and acceleration in the axial current: density operator vs Wigner function, JHEP 02 (2019) 146 [arXiv: 1807.03584] [INSPIRE].

[45] A. Vilenkin, Quantum Field Theory At Finite Temperature In A Rotating System, Phys. Rev. D 21 (1980) 2260 [inSPIRE].

[46] D.E. Kharzeev, J. Liao, S.A. Voloshin and G. Wang, Chiral magnetic and vortical effects in high-energy nuclear collisions-A status report, Prog. Part. Nucl. Phys. 88 (2016) 1 [arXiv: 1511.04050] [INSPIRE].

[47] M. Buzzegoli, Thermodynamic equilibrium of massless fermions with vorticity, chirality and electromagnetic field, Springer Cham (2020) [DOI]. 
[48] F. Becattini, Polarization in relativistic fluids: a quantum field theoretical derivation, (2020) [DOI].

[49] F. Becattini, V. Chandra, L. Del Zanna and E. Grossi, Relativistic distribution function for particles with spin at local thermodynamical equilibrium, Annals Phys. 338 (2013) 32 [arXiv: 1303.3431] [INSPIRE].

[50] S. Weinberg, Feynman Rules for Any Spin, Phys. Rev. 133 (1964) B1318 [InSPIRE].

[51] S. Weinberg, Feynman Rules for Any Spin. 2. Massless Particles, Phys. Rev. 134 (1964) B882 [INSPIRE].

[52] N.E. Nørlund, Vorlesungen über Differenzenrechnung, Springer (1924) [DOI].

[53] Y.L. Luke, The special functions and their approximations, Math. Sci. Eng. 53-A (1969) 1. 